\title{
Osteoglycin, a novel coordinator of bone and glucose homeostasis
}

\author{
N.J. Lee ${ }^{1,4}$, N. Ali ${ }^{2}$, L. Zhang ${ }^{1,4}$, Y. Qi ${ }^{1}$, I. Clarke ${ }^{1}$, R.F. Enriquez ${ }^{1,2}$, M. Brzozowska ${ }^{2,4}$, I.C. Lee ${ }^{1}$, \\ M.J. Rogers ${ }^{2,4}$, D.R. Laybutt ${ }^{3}$, J.R. Center ${ }^{2,4}$, P.A. Baldock ${ }^{2,4,5,6}$, H. Herzog ${ }^{1,4,5, *, 6}$
}

\section{ABSTRACT}

Objective: The skeleton, which is strongly controlled by endocrine factors, has recently been shown to also play an active endocrine role itself, specifically influencing energy metabolism. However, much less is known about this role. Therefore, we sought to identify novel endocrine factors involved in the regulation of both bone mass and whole-body glucose homeostasis.

Methods: We used transcriptomic and proteomic analysis of $Y 1$ receptor deficient osteoblasts combined with the generation of a novel osteoglycin deficient mouse model and performed comprehensive in vivo phenotype profiling, combined with osteoglycin administration in wildtype mice and human studies.

Results: Here we identify a novel role for osteoglycin, a secreted proteoglycan, in coordinating bone accretion with changes in energy balance. Using an osteoglycin knockout mouse model, we show that at a whole body level, osteoglycin acts to suppress bone formation and modulate whole body energy supplies by altering glucose uptake through changes in insulin secretion and sensitivity, as well as by altering food intake through central signaling. Examining humans following gastric surgery as a model of negative energy balance, we show that osteoglycin is associated with $\mathrm{BMl}$ and lean mass as well as changes in weight, BMl, and glucose levels.

Conclusions: Thus, we identify osteoglycin as a novel factor involved in the regulation of energy homeostasis and identify a role for it in facilitating the matching of bone acquisition to alterations in energy status.

(C) 2018 The Authors. Published by Elsevier GmbH. This is an open access article under the CC BY-NC-ND license (http://creativecommons.org/licenses/by-nc-nd/4.0/).

Keywords Bone; Osteoglycin; Neuropeptide Y; Osteoblasts; Glucose homeostasis

\section{INTRODUCTION}

The regulation of energy homeostasis is a complex process that must guarantee whole body energy requirements while ensuring adequate energy supply to individual tissues. In addition, changes in energy balance often reflect changes in body weight, and this presents an additional layer of complexity for weight-bearing tissues such as the skeleton. In particular, where positive energy balance precedes an increase in weight, bone must increase its strength to match the increasing mechanical demand. Inadequate adaptation would result in fracture and uncertain survival. While it is known that bone adapts to increasing body weight through mechanical stimuli, the mechanism whereby bone cells ensure adequate energy for adaptation during weight gain and whether non-mechanical triggers for adaptation exist are unknown.

NPY is an important regulator of energy homeostasis and has developed considerable complexity in order to coordinate the demands of energy balance at the organism- and tissue-specific level, playing a pivotal role within the central nervous system as well as being directly involved in numerous organ systems [1]. NPY circuits originating from the hypothalamus not only control energy intake but also play a particularly important role in coordinating whole body energy expenditure through peripheral energy partitioning, ensuring that specific organ systems receive the energy they require to function without risk to the organism as a whole. Bone is one system under substantial control by NPY signaling, acting from the brain as well as locally within the cells of bone itself $[2,3]$, consistent with its necessary ties to overall energy balance and body weight. Under conditions of negative energy balance, when hypothalamic NPY levels are high, NPY exerts a strong inhibitory tone on bone formation [4]. This suppression of bone production during periods of starvation provides a mechanism to match skeletal homeostasis to available energy stores and body weight, thereby protecting whole body energy reserves from depletion. Conversely, during positive energy balance NPY signaling decreases, releasing the inhibition of bone formation and increasing bone mass as body weight and its associated mechanical demand on the skeleton increases [4]. The central NPY axis acts through its Y2 receptors in the hypothalamus [2]. However, in addition to the actions of central NPY/Y2 receptors, in recent years the actions of peripheral $Y 1$ receptors has suggested the existence of a parallel axis, involving local production of

\footnotetext{
${ }^{1}$ Neuroscience Division, Garvan Institute of Medical Research, Darlinghurst, NSW, Australia ${ }^{2}$ Bone Biology Division, Garvan Institute of Medical Research, Darlinghurst, NSW, Australia ${ }^{3}$ Diabetes and Metabolism Division, Garvan Institute of Medical Research, Darlinghurst, NSW, Australia ${ }^{4}$ St Vincents Clinical School, UNSW Sydney, Sydney, NSW, Australia ${ }^{5}$ Faculty of Medicine, UNSW Sydney, Sydney, NSW, Australia

${ }^{6}$ P.A. Baldock and H. Herzog contributed equally to this work.

*Corresponding author. E-mail: h.herzog@garvan.org.au (H. Herzog).
}

Received March 21, 2018 • Revision received May 2, 2018 • Accepted May 4, 2018 • Available online 8 May 2018 
NPY and direct Y1 receptor signaling within bone forming osteoblasts. This was confirmed by the demonstration of increased bone formation and bone mass in osteoblast-specific $\mathrm{Y} 1$ receptor deletion models $[3,5]$ and decreased bone formation and mass in an osteoblast-specific NPY over expression model [6]. The local production and signaling of NPY in the osteoblast now suggests that, in parallel to the central circuit, a local energy-responsive circuit also exists that is critically involved in the coordination of bone-specific energy requirements.

Work from our laboratory has now progressed these investigations to show that NPY signaling via osteoblastic Y1 receptors not only controls bone mass but also contributes significantly to the control of wholebody insulin secretion and glucose homeostasis in mice through the release of novel factor(s), which are different from the previously implicated osteocalcin [7]. Specifically, a mouse model with conditional deletion of $\mathrm{Y} 1$ receptors in early osteoblasts, while displaying the expected high bone mass phenotype, also revealed impaired glucose tolerance, associated with reduced pancreatic islet volume and insulin secretion [7]. These glycemic changes were not evident when Y1 receptors were deleted from more mature osteoblasts [7], indicating a pathway confined to early cells of the osteoblast lineage. Analysis of bone marrow chimeras as well as in vitro experiments confirmed that the alterations in glucose and insulin homeostasis were the result of a direct endocrine signaling pathway originating in the osteoblast [7]. This represented the first mechanistic evidence for peripheral coordination of energy utilisation within bone tissue via an NPY/energy balance-dependent pathway; however, the capabilities and mechanism of such an axis were not known.

Here, we identify osteoglycin as a down-stream mediator of $\mathrm{Y} 1 \mathrm{re}$ ceptor signaling in early osteoblasts and its involvement in the communication between bone, energy and glucose homeostasis. We define novel actions for osteoglycin in the control of bone production, as well as in the modulation of whole body energy balance through the control of food intake and glucose uptake. To determine the exact role of osteoglycin in the co-ordination of bone, glucose, and whole body energy homeostasis, we have generated a novel osteoglycin deficient mouse model and performed comprehensive in vivo phenotype profiling, combined with osteoglycin administration in wildtype mice, human studies, and transcriptomic and proteomic analysis of $\mathrm{Y} 1$ receptor deficient osteoblasts.

\section{METHODS}

\subsection{Mice}

All animal experiments were approved by the Garvan Institute/St Vincent's Hospital Animal Experimentation Ethics Committee and conducted in accordance with relevant guidelines and regulations. All data presented are for male mice randomly assigned to experimental groups. Mice were group housed unless otherwise stated under a controlled temperature of $22{ }^{\circ} \mathrm{C}$ and a $12 \mathrm{~h}$ light cycle (lights on from 07:00 to 19:00 h) with ad libitum access to water. Mice were fed either a standard chow diet $(6 \%$ calories from fat, $21 \%$ calories from protein, $71 \%$ calories from carbohydrate, $2.6 \mathrm{kcal} / \mathrm{g}$, Gordon's Specialty Stock Feeds, Australia) or a high fat diet (HFD; 43\% kilojoules from fat, $17 \%$ kilojoules from protein, $40 \%$ kilojoules from carbohydrate, $4.8 \mathrm{kcal} / \mathrm{g}$, Specialty Feeds, Australia) starting from 7 weeks of age.

To isolate purified populations of osteoblasts, mice with green fluorescent protein (GFP) expression under control of a $3.6 \mathrm{~kb}$ fragment of the rat $\alpha 1(\mathrm{I})$-collagen promoter [8] were bred with previously described germline $\mathrm{Y} 1$ receptor knockout mice $\left(\mathrm{Y} 1^{-/-}\right)$[9]. GFP expression was confirmed using fluorescent microscopy of tails as well as PCR using oligos to detect the GFP transgene (forward oligo: $5^{\prime}$ GTTCTGCTGGTAGTGGTCG-3'; reverse oligo: 5'-CTGCACCACCGGCAAGCT GC- $3^{\prime}$ ). PCR was performed with 40 cycles with an annealing temperature of $58{ }^{\circ} \mathrm{C}$.

Mice with osteoblastic-specific deletion of the $\mathrm{Y} 1$ receptor $\left(\mathrm{Y}^{\mathrm{lox}}\right.$ ${ }^{10 x} 3.6 \mathrm{Cre}$ ) were generated and bred as previously described [7]. C57BL/ 6JAusb mice were sourced from the Mouse Engineering Garvan/ABR (MEGA) Facility (Moss Vale and Sydney, Australia). Mice expressing GFP under control of the NPY promotor (Tg (Npy-hrGFP)1Lowl) were obtained from The Jackson Laboratory (Bar Harbor, Maine, USA).

$\mathrm{Ogn}^{-1-}$ mice were produced by the Mouse Engineering Garvan/ABR (MEGA) Facility (Moss Vale and Sydney, Australia) by CRISPR/Cas9 gene targeting in C57BL/6J mouse embryos following established molecular and animal husbandry techniques [10]. To minimize the possibility of off-target genome modifications, the double-nicking approach utilizing the single strand-cleaving mutant of the Cas9 endonuclease (Asp10Ala $=$ Cas9n) was employed [11]. For this purpose, paired single guide RNAs (sgRNAs) were designed to target within the first coding exon (Exon 2) of Ogn. The sites used were TGTGAGTCCAGCTGCGACTGTGG and GTTAACTATGAGTATGCAACAGG (protospacer-associated motifs = PAMs underlined). To target Ogn, a solution consisting of the two sgRNAs $(15 \mathrm{ng} / \mu \mathrm{l}$ each) and full length, polyadenylated Cas9n mRNA (30 ng/ $\mu \mathrm{l})$ was prepared and microinjected into the nucleus and cytoplasm of C57BL/6J zygotes. Microinjected embryos were cultured overnight and those that underwent cleavage introduced into pseudo-pregnant foster mothers. Pups were screened by PCR across the two target sites and Sanger sequencing to detect those with modifications to Ogn. A founder male carrying a $21 \mathrm{bp}$ deletion within Exon 2 (Supplementary Figure 1) was identified, backcrossed to a wildtype C57BL/6J male, and progeny heterozygous for the deletion were inter-crossed to obtain homozygous $\mathrm{Ogn}^{-1-}$ mice.

\subsection{Human studies}

We conducted a prospective, non-randomized study of severely obese male and female patients undergoing three different weight loss interventions: dietary program, sleeve gastrectomy, and Roux-en-Y gastric bypass. The study was approved by the St Vincent's Hospital (Sydney, NSW) Human Research Ethics Committee in accordance with national guidance and all study participants signed written consent. The subjects were recruited in Sydney from Obesity Clinics at Royal Prince Alfred and Royal North Shore Hospitals as well as from private bariatric clinics at St George Private and St Vincent's Hospitals between October 2009 and May 2012. Inclusion criteria for the study were age between 18 and 70 years, a body mass index (BMI) $>35$ or $\mathrm{BMI}>30$ with medical complications from obesity. Women were either premenopausal or at least 5 years post menopause to avoid confounding by menopausal bone loss. Exclusion criteria were pregnancy and/or use of bone active (e.g. anti-resorptive) therapy. 22 patients who fulfilled the inclusion criteria were included in this study. The diet group underwent a weight loss program (Optifast ${ }^{\circledR}$ VLCD ${ }^{\mathrm{TM}}$ Program) under the care of their treating physician and dietician. Surgical procedures were performed according to currently accepted surgical techniques [12-14].

\subsection{Cell culture}

Bone marrow stromal cells (BMSCs) were isolated from male mice as previously described [3] using osteogenic media (control media supplemented with $50 \mathrm{mg} / \mathrm{l}$ ascorbic acid and $10 \mathrm{mM} \beta$-glycerophosphate). Briefly, following sacrifice by cervical dislocation, marrow was flushed from femurs and tibias with osteogenic media and cells were plated at a density of $1.9 \times 10^{6} \mathrm{cells} / \mathrm{cm}^{2}$ in $50 \mathrm{~cm}^{2}$ plastic tissue 
culture plates. The majority of non-adherent cells were removed by medium changes 3 and 5 days later. After 7 days in culture, cells were either collected for FACS sorting or trypsinized, counted, and replated at $3 \times 10^{4}$ cells $/ \mathrm{cm}^{2}$ in 24 -well plates in control medium. Cells were changed into differentiation medium 2 days later, and the medium then was changed three times per week until the cells were harvested. MIN6 cells were maintained in DMEM media containing $25 \mathrm{mM}$ glucose, 10\% FBS, $10 \mathrm{mM}$ HEPES, $100 \mathrm{U} / \mathrm{mL}$ penicillin, and $100 \mu \mathrm{g} /$ $\mathrm{mL}$ streptomycin. For treatment experiments, cells were seeded at $4 \times 10^{5}$ cells $/ \mathrm{mL}$, left to recover for $24 \mathrm{~h}$ and then media was changed to DMEM low glucose media $(5.5 \mathrm{mM})$ and $1 \%$ FBS for $24 \mathrm{~h}$ prior to treatment. MIN6 cells were treated for $4 \mathrm{~h}$ with recombinant mouse osteoglycin (R\&D Systems) at the stated doses after which RNA was extracted from the cells or the cells were subjected to a glucose stimulated insulin secretion (GSIS) test as described previously [7].

\subsection{Microarray analysis}

To obtain GFP-positive osteoblastic cells, BMSC cells were trypsinized after 7 days in culture, washed in $2 \%$ FBS in $1 \times$ PBS, strained through a $70 \mu \mathrm{m}$ strainer, pelleted by centrifugation and resuspended in FACS sorting buffer (1× PBS, 1 mM EDTA, 25 mM HEPES pH 7.0, 1\% FBS). GFP-positive osteoblasts were then collected by cell sorting using a FACS Vantage SETM (BD Biosciences) with FACSDiva SE ${ }^{\mathrm{TM}}$ software version 4.1.2 (BD Biosciences) using a nozzle size of $70 \mu \mathrm{m}$ at $4{ }^{\circ} \mathrm{C}$. RNA was extracted from GFP-positive osteoblastic cells using a Qiagen RNeasy Micro Kit (Qiagen) according to the manufacturer's instructions. RNA samples were subsequently checked for quality and quantified using the Agilent 2100 Bioanalyzer (Agilent Technologies) according to the manufacturer's instructions. Labeling and hybridization to an Affymetrix Mouse Gene 1.0 ST Array was performed by the Ramaciotti Centre for Gene Function Analysis (University of New South Wales, Sydney, Australia). Six arrays were performed on 4 separate wildtype samples and 2 separate $\mathrm{Y}^{-l-}$ samples.

Normalization and probe set summarization was performed using the robust multi-chip average [15] implemented in the Affy library [16] from R/Bioconductor $[17,18]$. Control probe sets were removed leaving 28,815 probe sets on the array. Differential gene expression was assessed for each probe set using Limma [19]. Gene Set Enrichment Analysis [20] was run with the GenePattern tool GSEApreranked using a ranked list of $t$-statistics derived from Limma analysis against c2_all, c3_all and c5_bp of the Molecular Signatures Database (MSigDB) [20]. All analyses were performed using Gene Pattern software [21] which is available at http://pwbc.garvan.unsw.edu.au/gp. Microarray data has been deposited in NCBI Gene Expression Omnibus (accession ID GSE114469).

\subsection{Mass spectrometry}

Proteins were extracted from BMSC cells that were cultured as described above for 14 days in osteogenic media in 6 well plates. $80 \mu \mathrm{g}$ of protein from 3 biological replicates of each genotype were resuspended in Lammeli buffer and separated by $10 \%$ SDS-PAGE, and an in-gel digest performed as previously described [22]. Following digestion, peptides were extracted and desalted using in-house prepared C18 StageTips [23].

Peptides were separated by nano-LC using an Easy-Nano LC II HPLC and autosampler system (Thermo) and mass spectra were acquired on the Orbitrap Velos Pro mass spectrometer (MS) (Thermo). Peptides were delivered to the MS with a nano-C18 column $(75 \mu \mathrm{x} \sim 20 \mathrm{~cm}, 5$ $\mu, 200 \AA$ Magic, Michrom) and the reverse phase nano-eluent was subject to positive nano-flow electrospray analysis in an information dependent acquisition mode (IDA) (400 nl/min over $60 \mathrm{~min})$. In IDA mode, survey scans in the range $\mathrm{m} / \mathrm{z} 350-1750$ were acquired with lockmass at $\mathrm{m} / z 445$ enabled and a resolution of 60,000. Up to the 20 most abundant ions ( $>5,000$ counts) with charge states $>+2$ were sequentially isolated and further subjected to MS/MS fragmentation within the linear ion trap using collisionally induced dissociation (CID). MS/MS spectra were accumulated with an activation time of $30 \mathrm{~ms}$ at a target value of 30,000 ions. $\mathrm{m} / \mathrm{z}$ ratios selected for MS/MS were dynamically excluded for $30 \mathrm{~s}$.

\subsection{Mass spectrometry data processing}

The nanoLC-MS/MS raw files were processed with MaxQuant software (version 1.2.7.4) for data processing and protein identification. MaxQuant employs the Andromeda algorithm for database searching and identification [24]. Extracted peak lists were searched against the UniProtKB/Swiss-Prot Homo sapiens database (Uniprot_ Mouse_02_2012) containing 54,221 entries (including common contaminants) and a proportionally sized decoy database for false discovery rate (FDR) generation. The following search parameters were selected; fixed cysteine carbamidomethylation modification; variable methionine oxidation modification, variable protein $\mathrm{N}$-acetylation, variable phosphorylation of serine, threonine and tyrosine; minimum peptide length of 6 amino acids and up to 2 missed cleavages were allowed. The initial first search mass tolerance was $20 \mathrm{ppm}$ for precursor ions and 0.5 Da for fragment ions, match between run was enabled with default settings and Label Free Quantitation (LFQ) was enabled. The false discovery rate limited $1 \%$ for both protein and peptide identifications. Peptides with posterior error probability greater than $10 \%$ were removed and protein identification required a minimum of 1 unique peptide. For phosphopeptides, those exhibiting a phosphosite localization probability $(\mathrm{LP})>0.75$ were included in further analyses. LFQ quantitation was performed using the Perseus Analysis Software. Briefly, proteins were filtered so that an LFQ value was present in at least $3 / 6$ of the samples and the missing values filled by imputation via normal distribution. All intensity values were Log2 transformed and the ratios determined between genotype groups. Proteins that exhibited a fold change greater than $20 \%$ were selected for further analyses.

\subsection{Metabolic phenotyping of $\mathrm{Ogn}^{-/-}$mice}

Whole body lean mass, fat mass, bone mineral content (BMC) and bone mineral density (BMD) were measured in $\mathrm{Ogn}^{-1-}$ mice and their wildtype littermates anesthetised with isoflurane using a dedicated mouse dual X-ray absorbitometry (DXA) (Lunar Piximus II, GE Medical Systems, Madison WI) at 7 weeks of age. Mice were then either maintained on a standard chow diet or placed on a high fat diet and body weight was monitored weekly.

Glucose tolerance tests were performed on the mice at 11 weeks of age (unless otherwise stated) at 14:00 to $16: 00 \mathrm{~h}$. Briefly, the mice were fasted for $5 \mathrm{~h}$ before i.p. injection between 14:00 to 16:00 $\mathrm{h}$ of a $10 \%$ D-glucose solution $(1.0 \mathrm{~g} / \mathrm{kg}$ ) (Astra Zeneca, North Ryde, NSW, Australia). Blood samples were obtained from the tail tip at $0,15,30$, 60 , and 90 min after glucose injection and blood glucose levels were measured using a glucometer (AccuCheck II; Roche, New South Wales, Castle Hill, Australia). Serum was also separated, immediately frozen, and stored at $-20^{\circ} \mathrm{C}$ for later measurement of serum insulin using a RIA kit from Millipore (Millipore, Billerica, MA, USA). 7 days later, mice underwent an insulin tolerance test, in which they were again fasted for $4 \mathrm{~h}$ before i.p. injection between 14:00 to 16:00 $\mathrm{h}$ with insulin $0.5 \mathrm{lU} / \mathrm{kg}$ ) (Novo Nordisk Pharmaceuticals, Baulkham Hills, NSW, Australia). Blood glucose levels were measured at 0, 15, 30, 45, 60, and 90 min after insulin injection using a glucometer. 
At 13 weeks of age and at least 7 days following insulin tolerance tests, indirect calorimetry measurements were performed using the Promethion Metabolic Cage System (Sable Systems International, Las Vegas, NV, USA). Mice were single housed and acclimatized for $72 \mathrm{~h}$ in the Promethion system before data acquisition began. The Promethion multiplexed system has a 'home-like' environment with each cage containing standard bedding, a food hopper, water bottle, and an enrichment tube for body mass measurements, connected to load cells for continuous monitoring. Instrument control and data acquisition were performed according to the manufacturer's instructions. Raw data were processed using ExeData software (Sable Systems).

\subsection{Tissue collection}

Mice were injected with the fluorescent compound calcein $(15 \mathrm{mg} / \mathrm{kg}$; Sigma-Aldrich, St Louis, MO, USA) 2 and 7 days prior to tissue collection to enable subsequent calculation of bone formation rate. Body composition was again measured as above by DXA 3 days prior to tissue collection. At 16 weeks of age, mice were culled between 13.00 and $16.00 \mathrm{~h}$ by cervical dislocation and decapitation for collection of trunk blood. Serum was separated, immediately frozen and stored at $-20^{\circ} \mathrm{C}$. The white adipose tissue (WAT) depots (right side inguinal, retroperitoneal, epididymal (gonadal) and mesenteric), and skeletal muscle were removed, weighed, frozen on dry ice, and stored at $-80{ }^{\circ} \mathrm{C}$. Femurs, tibias and caudal vertebrae were excised, fixed overnight in $4 \%$ paraformaldehyde (PFA) in phosphate buffered saline (PBS) at $4{ }^{\circ} \mathrm{C}$, and then stored in $70 \%$ ethanol at $4{ }^{\circ} \mathrm{C}$ before undergoing processing.

\subsection{Osteoglycin treatment}

In order to investigate whether osteoglycin treatment regulates glucose metabolism, male C57BL/6JAusb (wildtype) mice at 11-12 weeks of age were fasted over night and then received an i.p. injection of either PBS or osteoglycin the next morning. Four hours later, mice were subjected to either a glucose tolerance test or an insulin tolerance test as outlined above. For the initial pilot study, mice received a dose of either $0.3 \mu \mathrm{g}$ or $3 \mu \mathrm{g}$ osteoglycin obtained from R\&D Systems Inc. (Minneapolis, MN USA) or $6 \mu \mathrm{g}$ or $30 \mu \mathrm{g}$ osteoglycin obtained from Shanghai Biotech BioScience \&Technology Co., Ltd. (Shanghai, China). All subsequent treatment experiments were performed using a $3 \mu \mathrm{g}$ dose of osteoglycin obtained from R\&D Systems Inc. Another group received an i.p. injection of either saline or insulin $(0.5 \mathrm{IU} / \mathrm{kg}$ ) (Novo Nordisk Pharmaceuticals, Baulkham Hills, NSW, Australia) and $10 \mathrm{~min}$ later quadriceps muscles were taken, snap frozen in liquid nitrogen and stored at $-80{ }^{\circ} \mathrm{C}$ for subsequent analysis via western blot. Indirect calorimetric measurements following osteoglycin treatment were obtained using a different set of C57BL/6JAusb mice. Mice were single housed and acclimatised in the Promethion System (Sable Systems) for $72 \mathrm{~h}$ as outlined above prior to any data acquisition. Mice were given an i.p. injection of either $3 \mu \mathrm{g}$ osteoglycin (R\&D Systems) or saline at 16:30 $\mathrm{h}$ after baseline data had been acquired from one complete dark cycle and the majority of a light cycle without interrupting data acquisition. Data were acquired and analyzed as described above.

\subsection{Analysis of cfos and NPY signaling following osteoglycin administration}

One group of adult mice expressing GFP under control of the NPY promotor (Tg (Npy-hrGFP)1Lowl; The Jackson Laboratory, Bar Harbor, Maine, USA) received an i.p. injection of either PBS or osteoglycin $(3 \mu \mathrm{g})$. A second group was given intracerebroventricular (icv) injection with either PBS or $1 \mu$ l osteoglycin (R\&D Systems). Briefly for the icv injection, the mouse was anesthetized and placed on a Kopf stereotaxic frame (David Kopf Instruments, Tujunga, CA, USA). A $0.5 \mathrm{~cm}$ skin excision was made along the midline to expose the bregma. A small hole was drilled through the skull and the dura above the surface of the brain. A $2 \mu \mathrm{l}$ Hamilton syringe was used to reach the desired target according to the injection coordinates (from bregma: $1 \mathrm{~mm}$ lateral, $0.34 \mathrm{~mm}$ posterior, and $2.5 \mathrm{~mm}$ deep (Franklin and Paxinos, 2014)). $1 \mu \mathrm{l}$ of osteoglycin was then injected into the lateral ventricle slowly in $2 \mathrm{~min}$. The needle was left in for another $10 \mathrm{~min}$ to prevent the reflux. $30 \mathrm{~min}$ after both i.p. and icv injections the animals were perfused with saline and then $4 \%$ paraformaldehyde (PFA). Brains were post fixed in 4\% PFA for $2 \mathrm{~h}$, then placed in $30 \%$ sucrose overnight and cut at $35 \mu \mathrm{m}$ on a cryostat. Subsequently, fluorescent immunohistochemistry was performed using an antibody against cfos (Santa Cruz Biotechnology, Dallas, Texas, USA). GFP (representing NPY) and cfos expression were visualized in brain sections with a fluorescent microscope.

\subsection{Western analysis}

In order to determine protein levels of Akt in the total and phosphorylated forms, western blot analysis was performed on quadriceps muscle samples as previously described [25]. Briefly, powdered muscle samples were resuspended in buffer (PBS, pH 7.5; 1\% nonident NP-40; $0.5 \%$ sodium deoxy-cholate; and $0.1 \%$ SDS), supplemented with protease and phosphatase inhibitors $(10 \mu \mathrm{g} / \mathrm{ml}$ phenylmethylsulfonyl fluoride, $10 \mu \mathrm{g} / \mathrm{ml}$ aprotinin, $10 \mu \mathrm{g} / \mathrm{ml}$ leupeptin, $1 \mathrm{mmol} / \mathrm{I} \mathrm{Na}_{3} \mathrm{VO}_{4}$, and $10 \mathrm{mmol} / \mathrm{l} \mathrm{NaF}$ ) and solubilized for $2 \mathrm{~h}$ at $4{ }^{\circ} \mathrm{C}$. Equal amounts of tissue lysate $(25 \mu \mathrm{g}$ protein) were resolved by SDS-PAGE, transferred to immobilon-P membranes (Millipore, Billerica, MA,USA) and immunoblotted with antibodies against phospho-Akt (Ser473) (pAkt (473)) and Akt (Cell Signaling Technology, Danvers, MA, USA), and glyceraldehyde 3-phosphate dehydrogenase (GAPDH) (Cell Signaling Technology, Danvers, MA, USA). Signals were visualized by ECL (Perkin-Elmer) and bands were quantitated by densitometry. Expressions of pAkt (Ser473) and Akt were normalized to GAPDH for controlling loading variation. The relative pAkt (Ser473) to Akt ratios of various treatment groups as a percentage of that of control group are presented.

\subsection{Pancreas histology}

Pancreatic tissues were fixed in 4\% PFA, embedded in paraffin, and sectioned at $5 \mu \mathrm{m}$. Immunohistochemistry was then performed using rabbit anti-insulin (Santa Cruz Biotechnology, Texas, USA; 1:500) and biotinylated goat anti-rabbit (Vector Laboratories, California, USA; $1: 250)$ antibodies and a Vectastain $A B C$ kit (Vector Laboratories, California, USA). Insulin-positive islet cell number and size were quantified from at least five sections per pancreas (each $60 \mu \mathrm{m}$ apart) using a camera attached to a Leica microscope (Leica Microsystems) and Osteomeasure software (Osteometrics, Georgia, USA). Total pancreatic surface was also measured using ImageJ software (National Institutes of Health, USA) and used to determine islet cell number per $\mathrm{mm}^{2}$ and $\beta$-cell area (area positive for insulin immunostaining divided by the total pancreatic surface).

\subsection{Plasma assays}

Serum glucose (cull samples only) was measured using a glucose oxidase assay kit (Trace Scientific, Clayton, Victoria, Australia), serum insulin was measured using a RIA kit from Millipore, and serum osteoglycin was measured using an ELISA kit from Cloud-Clone Corp (Houston, USA).

\subsection{Bone micro-computed tomography (micro-CT) and histomorphometry}

Micro-CT and histomorphometric analyses of femora were carried out as previously described [7] with minor adjustments as detailed here. 
Briefly, following fixation, left femora were cleaned of muscle and analyses of the bone were carried out by micro-CT with a Skyscan 1172 scanner and associated analysis software (Skyscan, Aartselaar, Belgium) as previously described. Analyses of the cortical bone were carried out in 229 slices $(1.0 \mathrm{~mm})$ selected 572 slices $(2.5 \mathrm{~mm})$ proximally from the distal growth plate resulting in calculations of the following parameters: total tissue area, bone area, marrow area, periosteal perimeter, cortical thickness, and polar moment of inertia (an index of strength). Analyses of the trabecular bone were carried out in 229 slices $(1.0 \mathrm{~mm})$ selected 114 slices $(0.5 \mathrm{~mm})$ proximally from the distal growth plate resulting in calculations of the following parameters: total tissue volume, bone volume, trabecular number, trabecular thickness, and trabecular separation.

The 3rd lumbar vertebrae were also scanned with a Skyscan 1172 scanner and analyzed with the associated Skyscan software. An elliptical region of interest was fitted through the trabecular bone offset from the growth plates at each end by 10 slices $(0.044 \mathrm{~mm})$. Analyses within the region of interest resulted in calculations of the following parameters: total tissue volume, bone volume, trabecular number, trabecular thickness and trabecular separation.

Bone histomorphometry was carried out on $5 \mu \mathrm{m}$ sagittal sections of the distal half of the left femur as previously described [26]. Briefly, fluorescence microscopy was used to calculate mineral apposition rate, mineralizing surface, and bone formation rate while osteoclast surface and osteoclast number were estimated using tartrate-resistant acid phosphatase-stained sections.

\subsection{RNA extractions and cDNA synthesis}

Unless otherwise stated, all RNA extractions were carried out using TRIzol ${ }^{\circledR}$ reagent (Sigma) according to the manufacturer's instructions. RNA extractions from the mid-shaft of femurs and tibias isolated from male mice were homogenised in $2 \mathrm{~mL}$ TRIzol ${ }^{\circledR}$ reagent using a Polytron homogeniser. RNA samples were subsequently checked for consistent quality and quantified using the Agilent 2100 Bioanalyzer (Agilent Technologies) according to the manufacturer's instructions. $1 \mu \mathrm{g}$ of total RNA was taken for cDNA synthesis with oligo (dT)20 and random hexamers using the SuperScript III First-Strand Synthesis System for reverse transcription-PCR (Invitrogen).

\subsection{Quantitative real time-PCR}

Quantitative real-time PCR was carried out using the Lightcycler 480 Probes Mastermix and Universal Probe Library assays on the Lightcycler 480 System (Roche). To control for variability in amplification due to differences in starting mRNA concentrations, $\beta$-actin was used as an internal standard. The relative expression of target mRNA was computed from the target Ct values and the housekeeper Ct value using the standard curve method (Applied Biosystems).

\subsection{Human methods}

Weight was measured to the nearest $0.1 \mathrm{~kg}$ on a digital scale (TANITA, Wedderburg), and height was measured to the nearest $0.1 \mathrm{~cm}$ with a scale-mounted stadiometer. BMI was calculated as weight in $\mathrm{kg} /$ (height in meters) ${ }^{2}$. Body composition with measurements of lean body mass $(\mathrm{kg})$ and fat mass $(\mathrm{kg})$ were performed by total body dual-energy X-ray absorptiometry (DXA) using a Prodigy densitometer (GE LUNAR, Madison, WI, USA) prior to surgery. All measurements were performed twice, with the patient alighting from the instrument between scans, and their average was used in the analyses.

Blood samples, drawn following an overnight fast, were analyzed for glucose and osteoglycin at baseline and 1 month post-surgery. Blood glucose was determined by the hexokinase method (Roche modular $\mathrm{P}$ analyser, Roche Diagnostics, Castle Hill, NSW, Australia), osteoglycin was measured by ELISA (Cloud-Clone Corp) with all samples run on the same plate.

\subsection{Quantification and statistical analysis}

All data are expressed as means \pm SEM and statistical details of experiments can be found in the figures and figure legends or in the results text. Differences between groups were assessed using Student's $t$ test, ANOVA followed by Bonferroni's multiple comparison post hoc test, or repeated measures ANOVA where appropriate. Correlation analyses were carried out using the Pearson correlation measure. Statistical analyses were performed with Prism, version 6 or 7 (GraphPad Software Inc, La Jolla, CA, USA). Two-tailed $p=0.05$ was taken to be statistically significant.

\section{RESULTS}

3.1. Osteoblastic Y1 receptor signaling modulates insulin secretion and insulin action via osteoglycin

While investigating the mechanisms of $\mathrm{Y} 1$ receptor signaling in early osteoblasts, we observed, in addition to altered bone mass, a significant alteration in glucose homeostasis involving impaired glucose tolerance and reduced pancreatic beta cell mass and insulin secretion [7]. We also demonstrated that the supernatant from primary wildtype osteoblastic cultures was able to induce expression of the insulin genes in pancreatic MIN6 cells but that this effect was absent in Y1deficient osteoblastic cultures [7], indicating the presence of a direct osteoblast-derived regulator of beta cell function. Importantly, the ability of osteoblastic $\mathrm{Y} 1$ receptor signaling to alter glucose homeostasis was shown to be independent of osteocalcin [7]. Therefore, in order to identify potentially novel factors that are participating in the crosstalk between bone and pancreas, we utilised a combination of microarray and mass spectrometry. Firstly, expression profiling using microarray was carried out on RNA from primary osteoblastic cultures produced from bone marrow stromal cells (BMSCs) from wildtype and germline Y1-deficient mice, which were isolated by fluorescent activated cell sorting utilising an osteoblastic GFP reporter driven by a $3.6 \mathrm{~kb}$ fragment of the $\alpha 1(\mathrm{l})$-collagen promotor [8] (Figure 1A-C). The data were analysed for changes in expression of proteins belonging to secretory pathways. Interestingly, one factor, osteoglycin, stood out with a significant 3.1-fold down regulation in expression in the $\mathrm{Y} 1$ deficient osteoblastic cells. This was confirmed by qPCR showing significantly reduced osteoglycin mRNA levels in both Y1-deficient cultured osteoblastic cells and in the femora of male osteoblast specific $\mathrm{Y} 1$ null $\left(\mathrm{Y}^{\text {lox/lox }} 3.6 \mathrm{Cre}\right.$ ) compared to wildtype mice (Figure 1D-E). In order to identify whether the down regulation in osteoglycin mRNA levels translated into protein changes, we then performed mass spectrometry on osteoblastic cells isolated from BMSC cultures from $y 1^{\text {lox/lox }} 3.6 \mathrm{Cre}$ mice and their wildtype littermates. When the data were analysed for secretary proteins, 15 proteins were found to have a greater than 2-fold up-regulation and 35 proteins showed a greater than 2-fold down-regulation in $Y 1^{10 x / 10 x} 3.6 \mathrm{Cre}$ osteoblastic cells (Supplementary Table 1). Of note, neither the level of osteocalcin nor lipocalin 2, proteins previously shown to signal from osteoblasts to regulate aspects of energy metabolism [27,28], were altered, indicating they are not under the direct control of $Y 1$ signaling. Importantly, when comparing this mass spectrometry data with the microarray analysis of Y1-deficient osteoblasts, osteoglycin was found to follow the same pattern in both analyses, with a 3.6-fold down-regulation in protein levels as well as a 3.1-fold down-regulated mRNA expression in Y1-deficient osteoblasts. Indeed, osteoglycin was the only secreted 
A

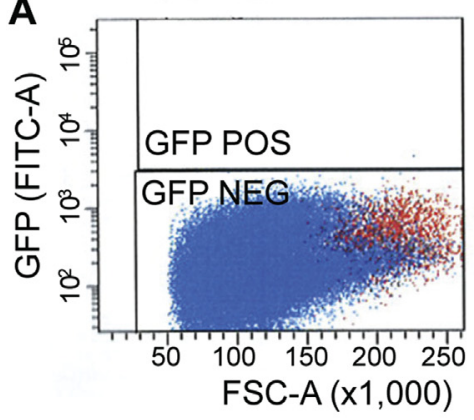

B

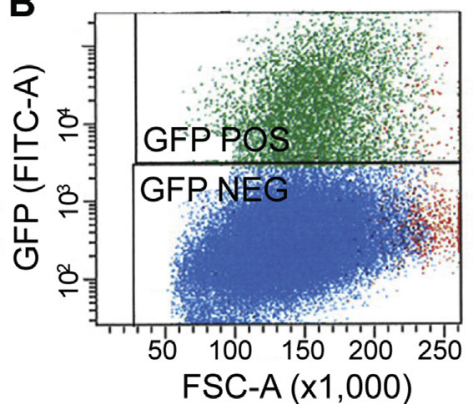

C

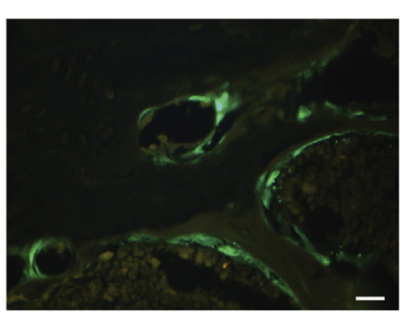

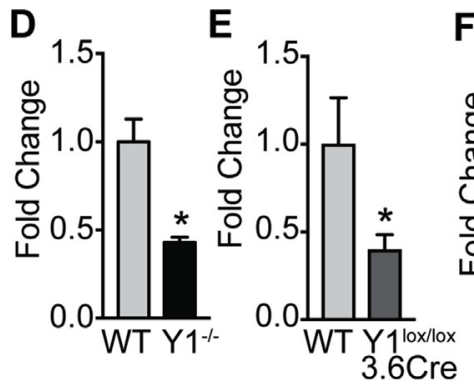
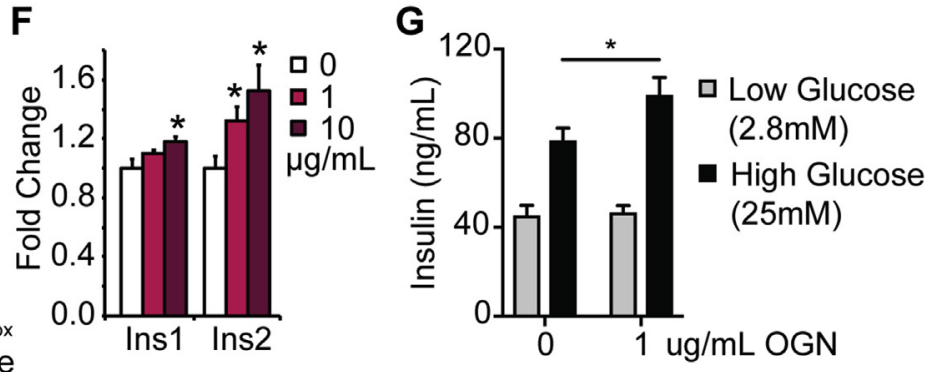

Figure 1: Osteoblastic Y1 receptor signaling modulates insulin secretion and insulin action via osteoglycin. Scatter plots showing osteoblast cells isolated from (A) non-GFP expressing mice and (B) GFP expressing mice showing GFP signal, forward scatter (FSC) and cell count. Green GFP positive (GFP P0S) gate represents the purified osteoblast cells that are selected for further analysis. (C) Representative photomicrograph showing GFP positive osteoblasts. qPCR of osteoglycin mRNA levels in (D) wildtype (WT) and $Y 1^{-1-}$ osteoblastic cells and (E) WT and Y1/ox/lox 3.6 Cre femora. (F) qPCR of Ins1 and Ins2 mRNA levels in MIN6 cells with different doses of osteoglycin. (G) Insulin secretion from MIN6 cells during a glucose stimulated insulin secretion test with or without osteoglycin $(1 \mu \mathrm{g} / \mathrm{mL})$. Data are means $\pm \mathrm{SEM}$ of at least 6 per group, ${ }^{*}=\mathrm{p}<0.05$ as indicated. Scale bar represents $5 \mu \mathrm{m}$.

protein that exhibited a greater than 2-fold change in the same direction in both microarray and mass spectrometric analyses. These data indicate that removing NPY signaling in early osteoblasts through deletion of the $\mathrm{Y} 1$ receptor results in a down-regulation of osteoglycin production, associated with reduced glucose tolerance and pancreatic insulin secretion, suggesting osteoglycin is a novel downstream mediator of osteoblastic $\mathrm{Y} 1$ receptor signaling that is important in the control of glucose homeostasis. To test whether osteoglycin is indeed a factor linking osteoblasts to insulin production, we used MIN6 cells to investigate the ability of osteoglycin to alter insulin gene expression. Treatment for $4 \mathrm{~h}$ with recombinant mouse osteoglycin resulted in a significant, dose-dependent increase in the expression of both Ins1 and Ins2 mRNA (Figure 1F). Furthermore, treatment with $1 \mu \mathrm{g} / \mathrm{mL}$ osteoglycin led to a significant increase in insulin secretion in a glucose stimulated insulin secretion assay (Figure 1G). This osteoglycin mediated increase in insulin expression and secretion in MIN6 cells is consistent with the reduction in insulin release seen in vivo in mice with reduced osteoglycin secretion from Y1 deficient osteoblasts.

\subsection{Osteoglycin controls glucose and insulin homeostasis}

In addition to bone, osteoglycin has been shown to be expressed in a variety of tissues including muscle and adipose tissue. While it is possible that these other sources of osteoglycin may also be contributing to the regulation of glucose metabolism, our original experiments using bone marrow chimeras to replace $\mathrm{Y} 1$ deficient osteoblasts with wildtype osteoblasts clearly demonstrated that this rescue can normalize the glucose homeostasis defect and confirmed the importance of bone derived osteoglycin as the critical component in this process [7]. However, in order to investigate the role of osteoglycin in glucose metabolism in a holistic sense, we used CRISPR technology to target the first coding exon of osteoglycin and generate osteoglycin deficient $\left(\mathrm{Ogn}^{-1-}\right)$ mice (Supplementary Figures $\left.1 \mathrm{~A}-\mathrm{B}\right)$. The resultant $O \mathrm{gn}^{-I-}$ mice showed no difference in either body weight or lean mass as measured by DXA (Supplementary Figures 1C-D); however, they did display a significant increase in the summed weight of dissected white adipose tissue depots (inguinal, epididymal, mesenteric and retroperitoneal) (Supplementary Figure 1E). In order to more precisely define the nature of osteoglycin's regulation of glucose metabolism and insulin action in vivo, we performed glucose tolerance tests on 11-12 week old $0 \mathrm{gn}^{-1-}$ mice and their wildtype littermates. $O \mathrm{gn}^{-1-}$ mice had a significant impairment in glucose tolerance under both chow and high fat diet (HFD) conditions (Figure 2A-B). Indeed, glucose levels in chow fed $\mathrm{Ogn}^{-1-}$ mice were similar to that of wildtype mice that had been fed a HFD for 5 weeks. Importantly, when $0 \mathrm{gn}^{-1-}$ mice were fed a HFD, there was a further marked impairment in glucose tolerance observed. Under both diets, the impairment in glucose tolerance was associated with elevated insulin levels, which was again more marked on HFD (Figure 2C-D) suggesting that the $\mathrm{Ogn}^{-1-}$ mice may be insulin resistant, which was further investigated by insulin tolerance tests. As shown in Figure $2 \mathrm{E}-\mathrm{F}$, glucose levels in $0 \mathrm{gn}^{-1-}$ mice in response to a dose of insulin remained higher compared to wildtype mice on the same diet, confirming that they were less responsive to insulin. We also measured serum glucose and insulin levels at cull at 16 weeks of age and found that chow-fed $\mathrm{Ogn}^{-1-}$ mice had significantly elevated glucose levels, similar to those of wildtype mice fed a high fat diet, although insulin levels were not significantly different (Figure 2G-H). Finally, although histological analysis showed no difference in the number of islets, it did reveal a significant reduction in total $\beta$-cell area in chow-fed (Figure 2I-J) but not HFD-fed (Supplementary Figures $1 \mathrm{~F}-\mathrm{G}$ ) $O g n^{-1-}$ mice compared to their wildtype littermates. The reduction in $\beta$-cell area seen in chow-fed $O \mathrm{gn}^{-I-}$ mice is similar to that seen in mice with selective deletion of the $\mathrm{Y} 1$ receptor in osteoblasts [7]. 

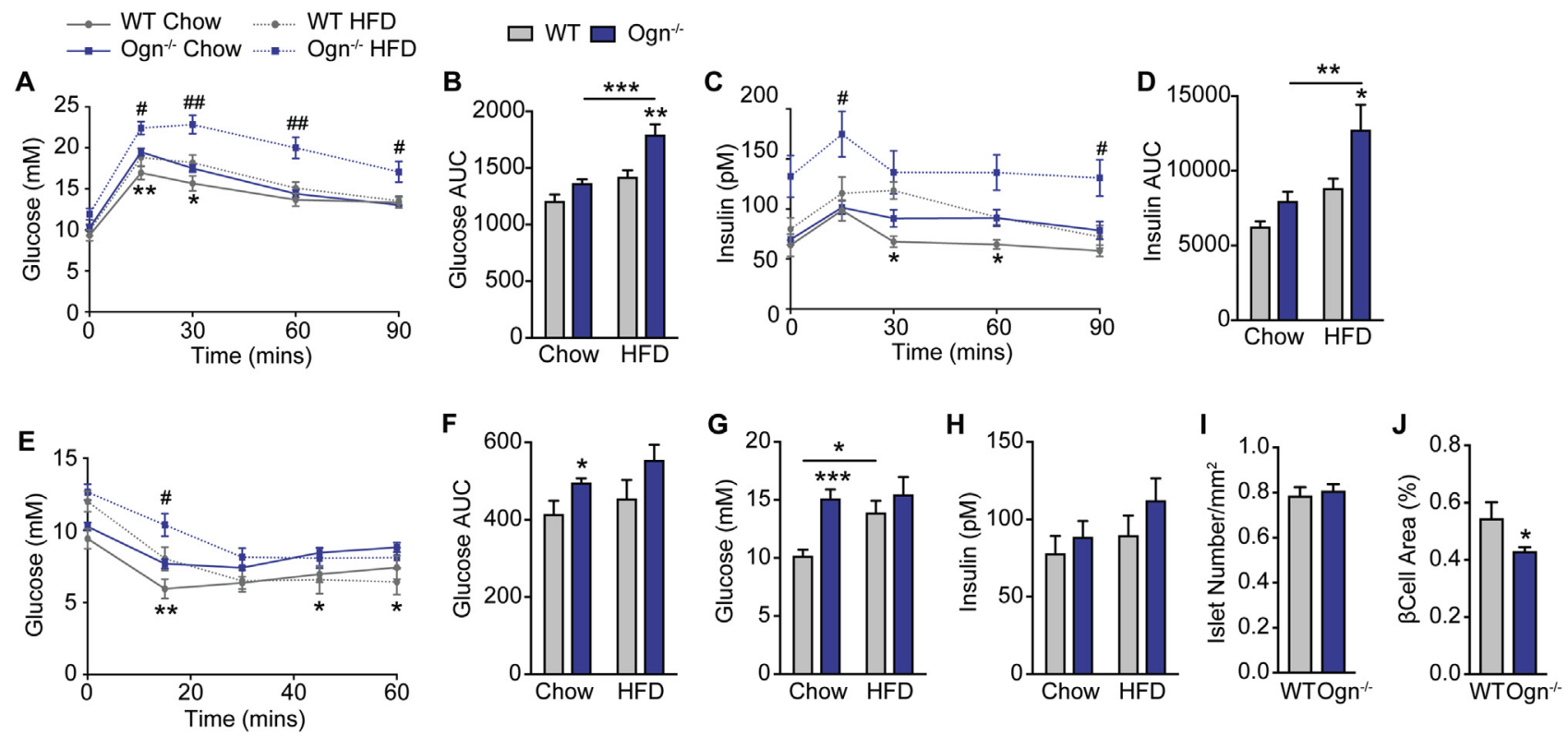

Figure 2: 0steoglycin deletion affects glucose and insulin homeostasis. $(A-B)$ Glucose and $(C-D)$ insulin levels during a glucose tolerance test shown either as a time course (A, C) or expressed as area under the curve (B, D) for $\mathrm{Ogn}^{-1-}$ and wildtype (WT) mice on both chow and high fat diet. (E) Glucose levels during an insulin tolerance test shown as a time course and (F) expressed as area under the curve for $\mathrm{Ogn}^{-1-}$ and WT mice on both chow and high fat diet. (G) Serum glucose and (H) serum insulin levels in Ogn ${ }^{-/-}$and WT mice on both chow and high fat diet at cull at 16 weeks of age. Histological analysis showing (I) number of islets and (J) beta cell area in the pancreas of 0 gn ${ }^{-/-}$and WT mice on chow diet. Data are means $\pm \mathrm{SEM}$ of at least 10 mice per group, except $4-6$ mice per group for islet histology $(\mathrm{I}-\mathrm{J})$. $^{\star}=\mathrm{p}<0.05,{ }^{\star \star}=\mathrm{p}<0.01,{ }^{\star \star \star}=\mathrm{p}<0.001$ between chow groups or as indicated; ${ }^{\#}=p<0.05,{ }^{\# \#}=p<0.01$ between HFD groups.

\subsection{Osteoglycin treatment improves glucose and insulin homeostasis}

As osteoglycin deficiency led to an impairment in glucose tolerance and insulin resistance, we sought to determine whether osteoglycin treatment could improve whole body glucose metabolism in vivo. To this end, we performed glucose tolerance tests in 14-15 weeks old wildtype mice either treated with or without osteoglycin $4 \mathrm{~h}$ prior to the glucose tolerance test. An initial dose response experiment was performed in which mice were treated with either PBS or a $0.3 \mu \mathrm{g}, 3 \mu \mathrm{g}$, $6 \mu \mathrm{g}$ or $30 \mu \mathrm{g}$ dose of osteoglycin $4 \mathrm{~h}$ prior to a glucose tolerance test. As shown in Figure $3 \mathrm{~A}$, osteoglycin treatment led to a dose dependent decrease in blood glucose levels during a glucose tolerance test. The $3 \mu \mathrm{g}$ dose was then used for a more detailed analysis of the effect of osteoglycin on glucose metabolism in vivo. Despite similar basal glucose and insulin levels before the osteoglycin injection, mice treated with osteoglycin had significantly lower glucose levels $4 \mathrm{~h}$ after osteoglycin treatment with no significant difference in insulin levels (Figure 3B-C). Furthermore, in response to a glucose bolus, mice treated with osteoglycin showed a lower blood glucose excursion and reduced area under the glucose curve compared to those of the control group (Figure 3D-E). Osteoglycin treatment also led to a lower blood glucose excursion during a glucose tolerance test in wildtype mice that had been fed a HFD for 3 weeks (Figure 3F). When osteoglycin was administered at the same time as a glucose bolus, no effect on glucose tolerance was observed (data not shown). Importantly, the improvement in glucose tolerance induced by prior treatment with osteoglycin in chow-fed wildtype mice was associated with a reduction in insulin levels throughout the glucose tolerance test compared to those of the PBS treated group (Figure 3G), suggesting that osteoglycin under an acute setting enhances insulin action and thus improves glucose tolerance. Therefore, we also performed insulin tolerance tests in a similar manner on wildtype mice either treated with or without osteoglycin $4 \mathrm{~h}$ prior. As shown in Figure $3 \mathrm{H}$, osteoglycin induced a significantly increased decline in glucose levels in response to insulin, confirming that osteoglycin is enhancing insulin action.

In order to identify the underlying mechanism behind osteoglycin's ability to enhance insulin action, we investigated its influence upon insulin stimulated AKT phosphorylation, a marker of glucose transport [29]. To test this, we determined the Akt phosphorylation response upon insulin stimulation (0.5 IU/kg, intraperitoneal (i.p.)) in the quadriceps muscle of wildtype mice treated with either PBS or osteoglycin $(3 \mu \mathrm{g}) 4 \mathrm{~h}$ prior to the insulin injection. As expected, insulin significantly increased the Akt phosphorylation in PBS-treated mice (Figure 3I-J). More importantly, this insulin-induced Akt phosphorylation was significantly enhanced in mice pre-treated with osteoglycin (Figure 3l$\mathrm{J})$, demonstrating a synergistic effect of insulin and osteoglycin signaling on glucose transport in vivo.

\subsection{Lack of osteoglycin leads to an increase in bone mass}

Since we identified osteoglycin in an osteoblast-specific mutant mouse with increased bone mass, we next investigated osteoglycin's effect upon bone homeostasis. DXA analysis on isolated femora from 16 week old wildtype and $O \mathrm{gn}^{-/-}$mice revealed that $\mathrm{Ogn}^{-/-}$mice have a significant increase in both femoral bone mineral density (BMD) and bone mineral content (BMC) as well as femur length (Figure $4 A-C$ ), confirming a critical role for osteoglycin in the regulation of bone mass. To further characterize this increase in bone mass, $\mu \mathrm{CT}$ analysis was performed. As shown in Figure 4D, mice lacking osteoglycin had a generalized increase in bone mass across the length of the femur and this was statistically significant when expressed as area under the curve (Figure 4E). Two specific regions in the distal femur (marked in Figure 4D) were analyzed in greater depth for changes in trabecular and cortical parameters, which showed that $0 g n^{-1-}$ mice have significantly greater trabecular bone volume (BV/TV) associated with an 
A

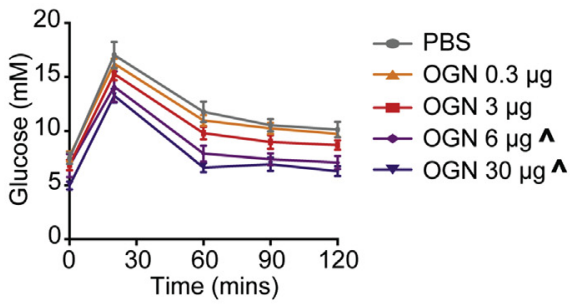

B

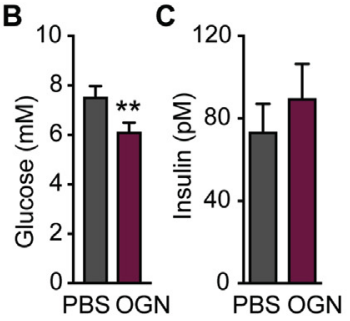

G

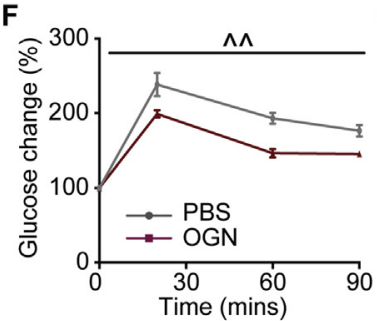

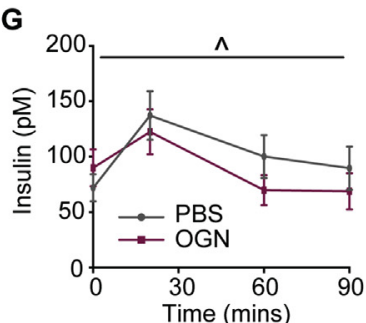

D
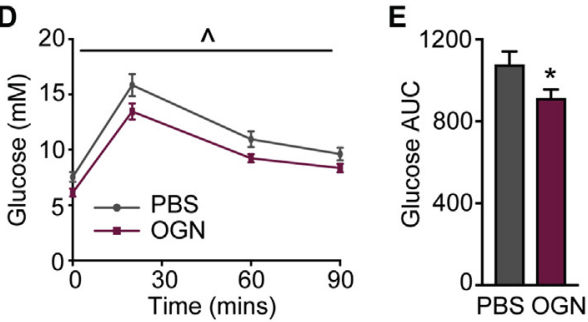

1

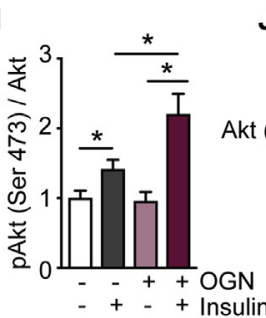

J

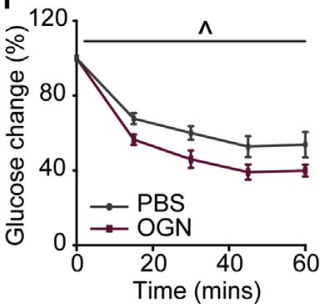

Insulin $\frac{\text { PBS }}{-+} \frac{\text { OGN }}{-+}$

ker473)

Akt

GAPDH

$\mathrm{kt}=$

Figure 3: Osteoglycin treatment controls glucose and insulin homeostasis. (A) Glucose levels during a pilot glucose tolerance test in WT mice treated with either PBS or different doses of osteoglycin $4 \mathrm{~h}$ prior to the test. (B) Glucose and (C) insulin levels in fasted WT mice $4 \mathrm{~h}$ after treatment with either osteoglycin or PBS. (D) Glucose levels during a glucose tolerance test and (E) expressed as area under the curve in chow-fed WT mice as well as (F) glucose levels during a glucose tolerance test in HFD-fed WT mice treated with either osteoglycin or PBS $4 \mathrm{~h}$ prior to the test. (G) Insulin levels during the glucose tolerance test in chow-fed WT mice treated with either osteoglycin or PBS $4 \mathrm{~h}$ prior to the test. (H) Glucose levels during an insulin tolerance test in WT mice treated with either osteoglycin or PBS $4 \mathrm{~h}$ prior to the test. (I-J) pAkt (Ser473) in quadriceps muscle of WT mice treated with osteoglycin or PBS followed by either saline or insulin $(0.5 \mathrm{lU} / \mathrm{kg}) 4 \mathrm{~h}$ later. (I) pAkt to Akt ratios of treatment relative to untreated group; (J) representative immunoblots. Data are means \pm SEM of at least 10 mice per group, except $>3$ mice per group for pilot study (A), and $4-6$ mice per group for pAkt experiment $(I-J)$. ${ }^{\star}=p<0.05,{ }^{\star \star}=p<0.01$ as indicated; ${ }^{\wedge}=\mathrm{p}<0.05,{ }^{\wedge}=\mathrm{p}<0.01$ OGN treatment effect over time compared to PBS as measured by repeated measures ANOVA.
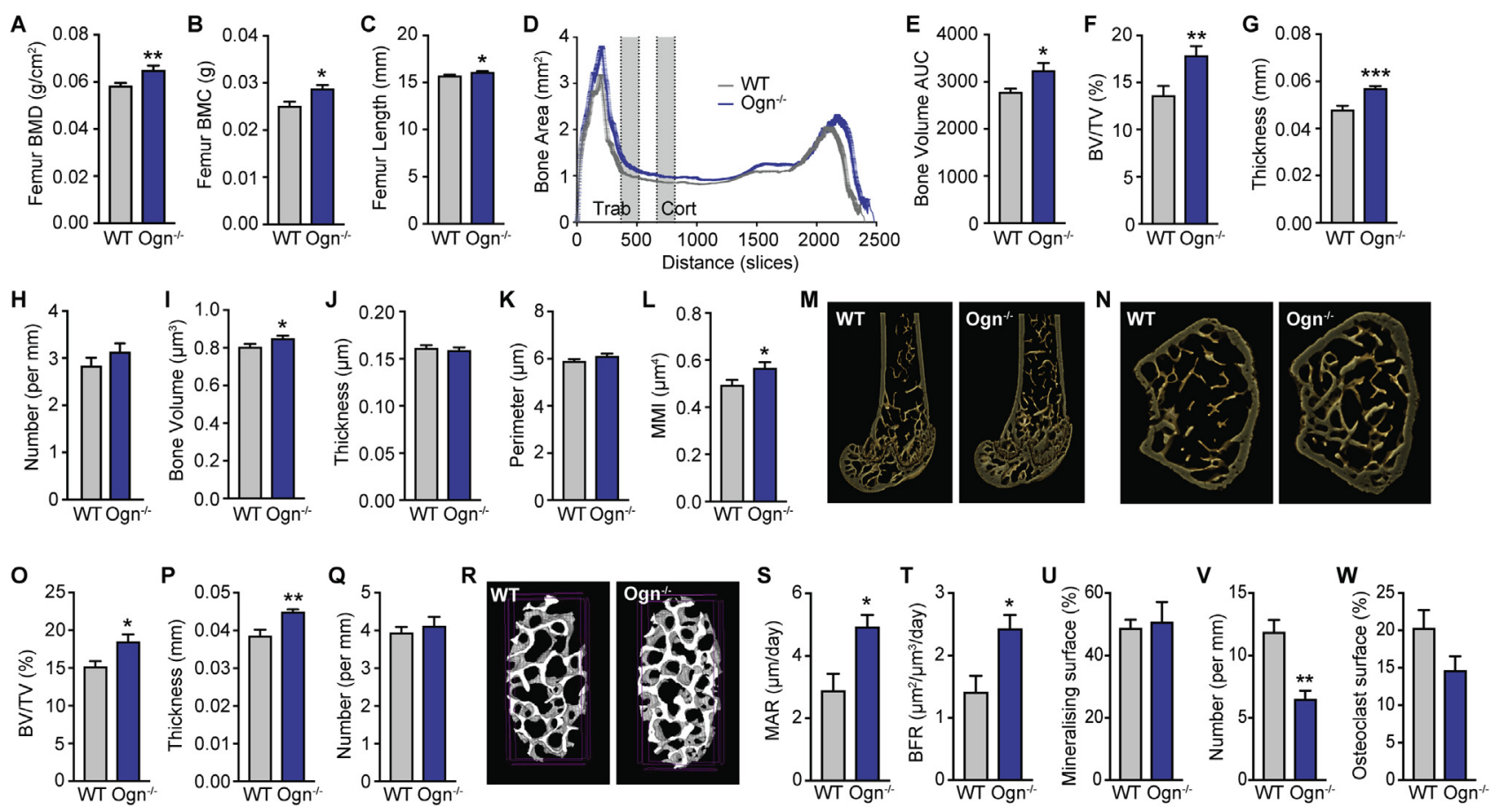

Figure 4: Lack of osteoglycin leads to an increase in bone mass. (A) BMD and (B) BMC of isolated femora from $0 \mathrm{gn}^{-1-}$ and wildtype (WT) mice as measured by DXA. (C) Femur length. (D) Bone area as measured by micro-CT across the length of femora also represented as (E) area under the curve. Micro-cT analysis of femoral trabecular bone showing (F) BV/TV, (G) trabecular thickness, and (H) trabecular number. Micro-cT analysis of femoral cortical bone showing (I) bone volume, (J) cortical thickness, (K) periosteal perimeter, and (L) mean polar moment of inertia (MMI). (M) Representative sagittal and (N) cross-sectional images from femora of $O \mathrm{gn}^{-1-}$ and WT mice. Vertebral micro-cT analysis of trabecular bone in the 3rd lumbar vertebrae showing (0) BV/TV, (P) trabecular thickness, (Q) trabecular number and (R) representative images. Histomorphometric analysis showing (S) mineral apposition rate (MAR), (T) bone formation rate (BFR), (U) mineralising surface, (V) osteoclast number, and (W) osteoclast surface in the femora of $0 \mathrm{gn}^{-1-}$ and WT mice. Data are means \pm SEM of at least $5-7$ per group, ${ }^{*}=p<0.05,{ }^{*}=p<0.01$ as indicated. 

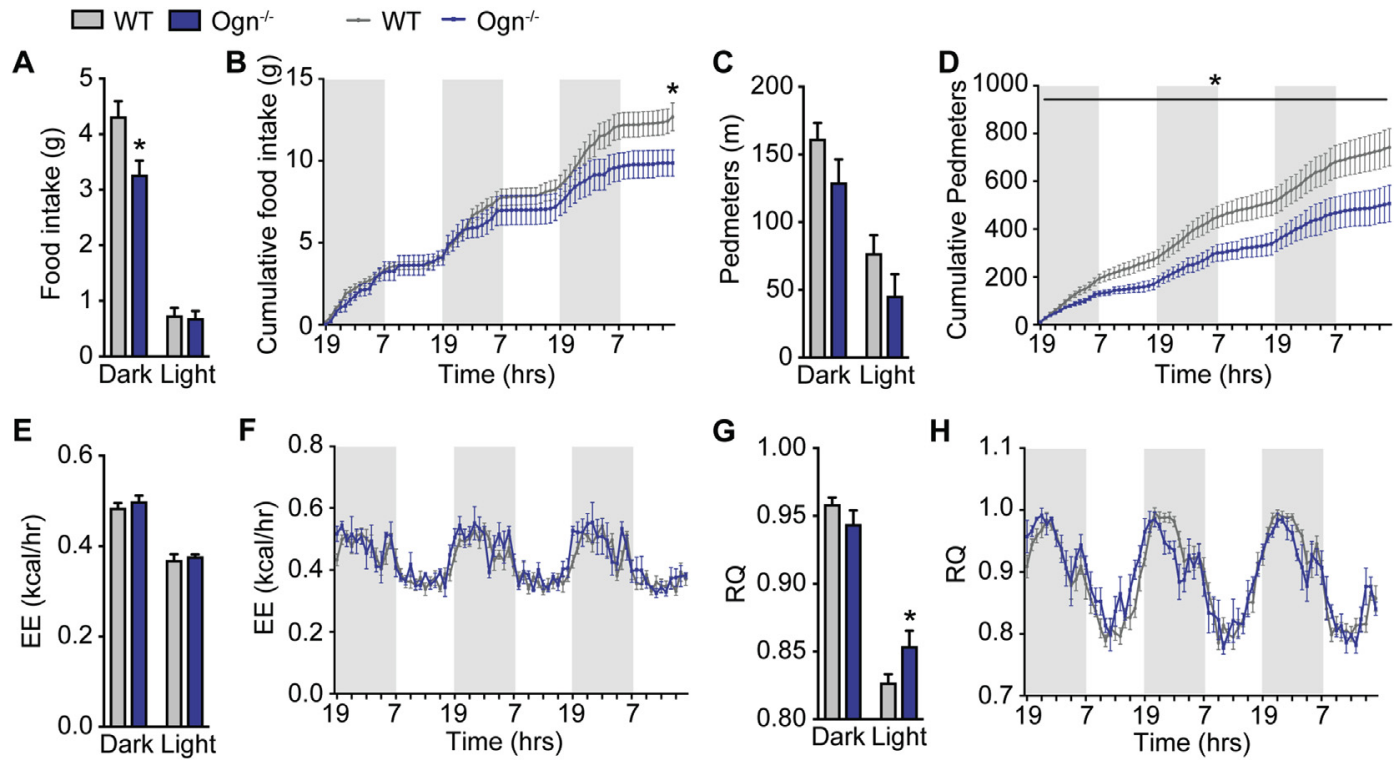

Figure 5: Lack of osteoglycin alters metabolic and activity parameters. (A) Averages for food intake during the dark and light phases, (B) cumulative food intake over a $72 \mathrm{~h}$ time course, $(C)$ averages for distance in voluntary locomotion travelled (pedmeters) during the dark and light phases, (D) cumulative pedmeters over a $72 \mathrm{~h}$ time course, (E) averages for energy expenditure during the dark and light phases, ( $F$ ) energy expenditure over a $72 \mathrm{~h}$ time course, $(\mathrm{G})$ averages for respiratory quotient (RQ) during the dark and light phases, and $(\mathrm{H}) \mathrm{RQ}$ over a $72 \mathrm{~h}$ time course in $0 \mathrm{gn}^{-1-}$ compared to wildtype (WT) mice. Data are means $\pm \mathrm{SEM}$ of at least 4 per group, ${ }^{*}=\mathrm{p}<0.05$ as indicated. Shaded regions represent dark phase.

increase in trabecular thickness but no change in trabecular number (Figure $4 \mathrm{~F}-\mathrm{H}$ ). Cortical bone volume was also significantly increased (Figure 4I) and, although there were no significant changes in cortical thickness or periosteal perimeter (Figure $4 \mathrm{~J}-\mathrm{K}$ ), the increase in cortical bone volume was associated with increased polar moment of inertia, an index of bone strength (Figure 4L). Furthermore, the increase in trabecular bone volume was also apparent in the vertebrae, as $\mu \mathrm{CT}$ analysis of the 3rd lumbar vertebrae revealed a similar significant increase in trabecular BV/TV and trabecular thickness with no change in trabecular number (Figure 40-R).

In addition, histomorphometric analysis revealed that the increase in bone mass evident in $\mathrm{Ogn}^{-/-}$mice was associated with a marked increase in osteoblast activity as shown by significantly enhanced mineral apposition rate (MAR) and bone formation rate (BFR), without a change in the amount of bone undergoing bone formation (mineralizing surface; Figure $4 \mathrm{~S}-\mathrm{U}$ ). In addition, there was a significant decrease in osteoclast number but no significant change in osteoclast surface (Figure 4V-W). Taken together, these data demonstrate that the increase in trabecular and cortical bone mass seen in $\mathrm{Ogn}^{-1-}$ mice is primarily a result of an imbalance in bone remodeling favoring increased osteoblast activity and bone formation and thus osteoglycin acts as a negative regulator of osteoblast activity.

\subsection{Osteoglycin promotes food intake}

A previous study has shown that i.p. injection of an osteoglycinmaltose binding protein complex inhibited food intake in mice associated with an upregulation of interleukin (IL)-1B and IL- 6 in the hypothalamus [30]. However, in that study, no significant differences in basic food intake between wildtype and $\mathrm{Ogn}^{-1-}$ mice were found [30]. Therefore, we assessed food intake, energy expenditure, and physical activity over a 3-day period using the Promethion metabolic and behavioral monitoring system. Interestingly, our $\mathrm{Ogn}^{-1-}$ mice displayed a significant decrease in food intake during the dark phase (Figure 5A), which continued over the entire monitoring period, resulting in a significant reduction in cumulative food intake over time (Figure 5B). In addition, $\mathrm{Ogn}^{-1-}$ mice displayed a trend towards reduced activity during both the dark and light phases (Figure $5 \mathrm{C}$ ), which was significant over time (Figure 5D). No significant difference was observed in absolute energy expenditure between $\mathrm{Ogn}^{-1-}$ and wildtype mice (Figure 5E-F) or when energy expenditure was normalized to body weight or lean mass (data not shown). However, $O \mathrm{On}^{-1-}$ mice had a significant increase in their mean respiratory quotient $(\mathrm{RQ}$ ) during the light phase (Figure $5 \mathrm{G}-\mathrm{H}$ ) suggesting that they burn less fat and more carbohydrate as fuel, data which are consistent with their increase in total white adipose tissue weight and greater blood glucose levels.

To further investigate the effect of osteoglycin on food intake, we next injected mice with osteoglycin $(3 \mu \mathrm{g})$ or PBS (i.p.) just prior to the onset of the dark phase while they were in the Promethion system. As shown in Figure 6A, food intake was similar between the two groups prior to treatment. However, osteoglycin treatment induced a transient increase in food intake, which was significant during the first half of the dark phase (Figure $6 \mathrm{~B}-\mathrm{C}$ ).

\subsection{Osteoglycin modulates central NPY to alter food intake}

To further explore how osteoglycin alters food intake and how this might be integrated into the overall regulation of whole body energy homeostasis, we investigated central alterations in response to osteoglycin changes. Firstly, a peripheral i.p. injection of osteoglycin in mice was able to significantly induce cfos expression in the arcuate nucleus (Arc) of the hypothalamus, confirming central involvement (Figure 6D-E). In order to determine whether this effect of osteoglycin on neuronal activation is direct or indirect, we next investigated cfos activation in the hypothalamus after intracerebroventricular (icv) injection of either osteoglycin or saline. As shown in Figure $6 \mathrm{~F}-\mathrm{G}$, icv treatment with osteoglycin induced strong expression of cfos in the Arc compared to control. As NPY is one of the most powerful central mediators of food intake, we performed this experiment in mice 
A

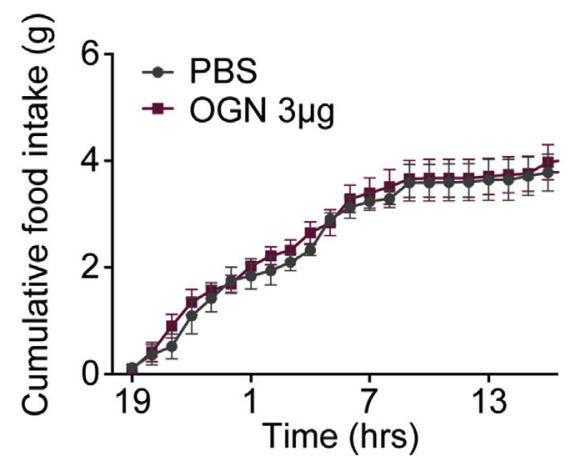

D
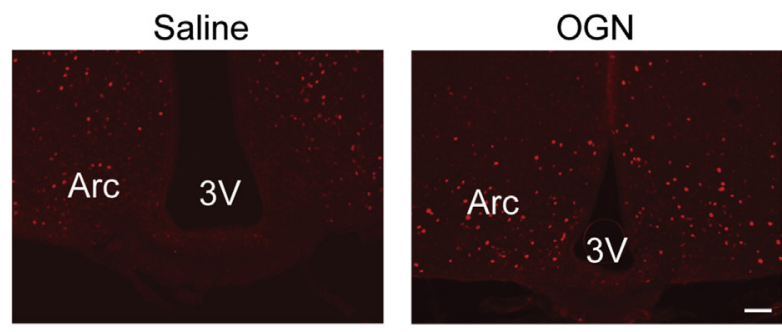

$\mathbf{F}$
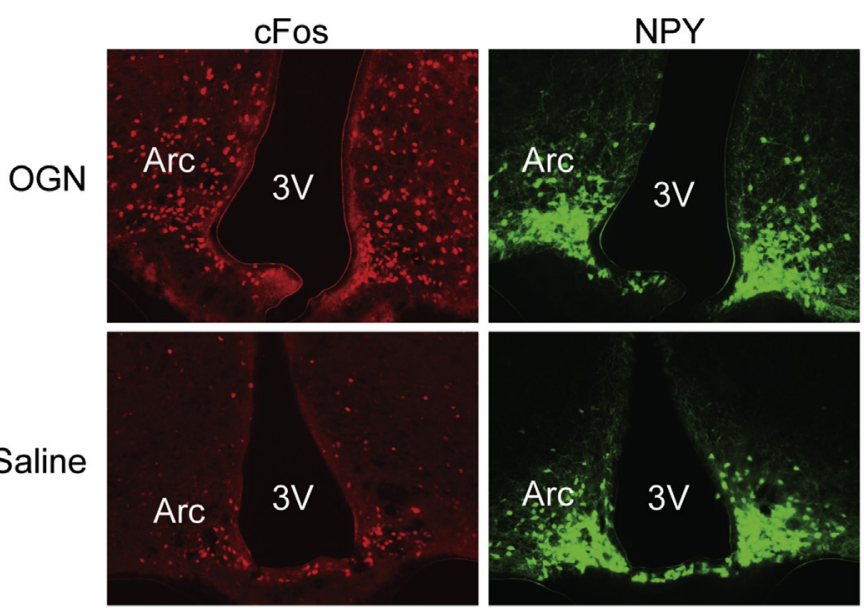

Saline

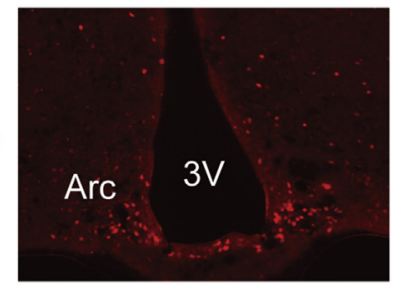

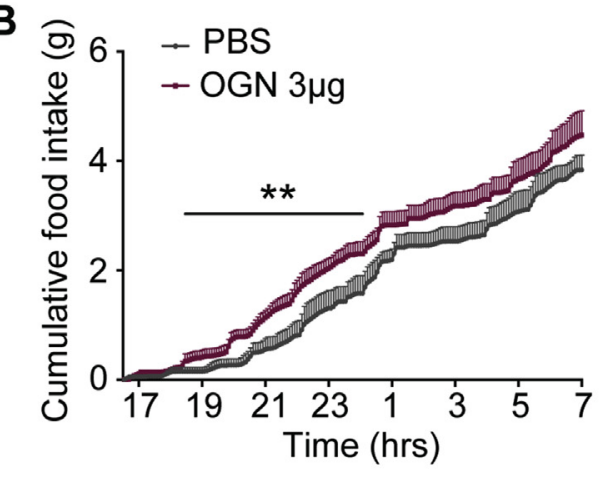

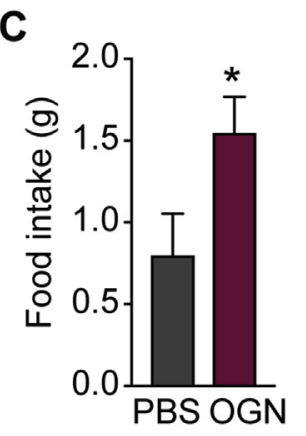

E

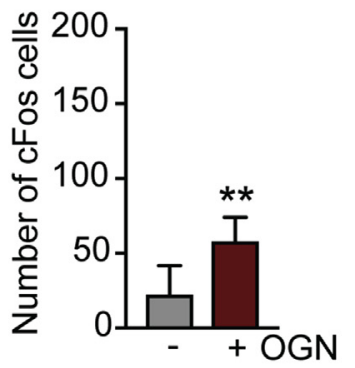

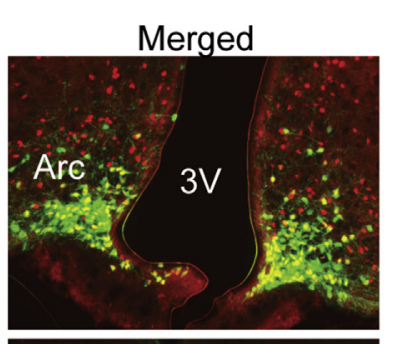

G
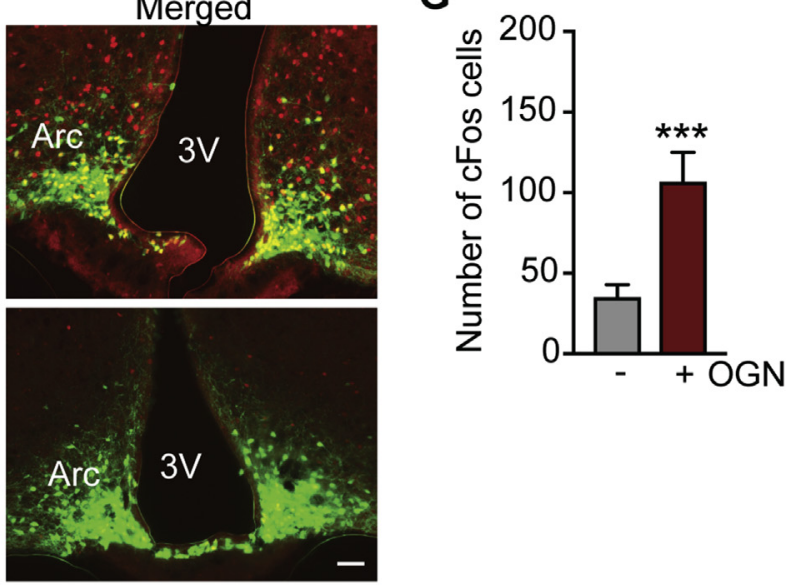

H

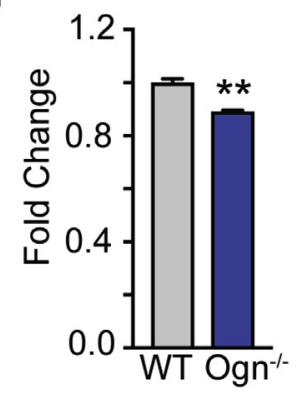

Figure 6: 0steoglycin modulates central NPY to alter food intake. Cumulative food intake in wildtype mice treated with either osteoglycin (OGN) or PBS control (A) prior to treatment and (B) immediately following treatment. (C) Total food intake during the first $6 \mathrm{~h}$ following OGN or PBS treatment. (D) Representative photomicrographs and (E) quantification showing cfos expression in the Arcuate nucleus of the hypothalamus following i.p. injections of either saline or OGN. (F) Representative photomicrographs and (G) quantification showing cfos (red), NPY (green) and cfos/NPY co-localization (yellow) expression in the Arcuate nucleus of the hypothalamus following icv injections of either saline or OGN. (H) qPCR of NPY mRNA levels in the Arcuate of WT and $0 g n^{-1-}$ brains. Data are means \pm SEM of at least 4 per group, ${ }^{\star}=p<0.05,{ }^{\star \star}=p<0.01,{ }^{\star \star \star}=p<0.001$ as indicated. Scalebar represents $100 \mu \mathrm{m}$. 
expressing GFP under control of the NPY promotor and thus were able to demonstrate that cfos expression in the Arc induced by osteoglycin significantly overlapped with NPY expression (Figure 6F; Percentage co-localization: osteoglycin $19.54 \% \pm 1.24$; saline $2.79 \% \pm 1.25$; $p<0.001$ ). In addition, analysis of hypothalamic NPY levels by qPCR revealed a significant downregulation of NPY mRNA in $\mathrm{Ogn}^{-1-}$ mice (Figure $6 \mathrm{H}$ ). This downregulation of hypothalamic NPY is consistent with the observed reduction in food intake in these mice and the stimulatory response to feeding by osteoglycin injection.

\subsection{Osteoglycin negatively correlates with fat and bone mass}

In order to more clearly define the relationship between osteoglycin and energy, glucose, and bone metabolism, we used a high fat diet to induce an increase in energy balance/obesity. High fat diet feeding in wildtype mice produced a significant increase in body weight, fat mass, and glucose intolerance (Supplementary Figures 2A-E). Interestingly, it also led to a significant decrease in circulating osteoglycin levels compared to mice on a chow diet (Figure 7A). Furthermore, serum osteoglycin significantly negatively correlated with body fat $\left(R^{2}=0.13, p<0.05\right)$ across the diet groups (Figure $7 B$ ), supporting a link between positive energy balance and reduced osteoglycin. Consistent with the disrupted insulin signaling and elevated circulating blood sugar seen in $\mathrm{Ogn}^{-1-}$ mice, serum osteoglycin displayed a highly significant negative relationship with both fasting glucose $\left(R^{2}=0.24, p<0.01\right)$ and insulin $\left(R^{2}=0.42, p<0.001\right)$ levels in these mice (Figure 7C-D). Finally, and indicative of a role for osteoglycin to suppress bone mass, serum osteoglycin levels were significantly negatively correlated with bone mass $\left(R^{2}=0.14, p<0.05\right)$ (Figure $7 \mathrm{E}$ ). It has been previously shown that high blood glucose favors bone formation through glut1 mediated upregulation of the key osteoblast transcription factor Runx2 in early osteoblasts [31], enhancing bone accretion and enabling the weight/bone adaptive process to proceed during obesity. Consistent with this, blood glucose and bone mass were also significantly correlated in these mice $\left(R^{2}=0.17, p<0.05\right)$ (Figure 7F).

Together, these data suggest that a decrease in serum osteoglycin promotes the adaptation of bone mass to the increase in body weight/ fat mass during high fat feeding. In order to further examine this relationship, we examined the skeletal consequences of high fat feeding in $\mathrm{Ogn}^{-1-}$ and wildtype mice. As expected, both genotypes exhibited increased body weight and fat mass on high fat diet compared to chow (Figure $7 \mathrm{G}-\mathrm{H}$ ), and, consequentially, cortical bone volume, periosteal perimeter, and $\mathrm{MMI}$ all increased in response to the additional mechanical loading associated with greater body weight (Figure $7 \mathrm{I}-\mathrm{L}$ ). However, a marked difference was observed in the trabecular bone response to high fat feeding between wildtype and $\mathrm{Ogn}^{-1-}$ mice. Consistent with the obesity-induced decrease in osteoglycin production, wildtype mice increased their bone mass towards that of $\mathrm{Ogn}^{-1-}$ mice when exposed to high fat feeding. This was due to the ability of fat-fed wildtype but not fat-fed $\mathrm{Ogn}^{-1-}$ mice to significantly increase their trabecular bone volume through increases in trabecular thickness compared to chow-fed controls (Figure $7 \mathrm{M}-\mathrm{N}$ ). This increase in trabecular bone volume seen in fat-fed wildtype mice compared to chow-fed controls was 3-fold greater than that observed in $\mathrm{Ogn}^{-1-}$ mice. This diet intervention study therefore identifies, for the first time, that bone-derived osteoglycin may be acting as a novel agent, co-ordinating the adaptive response between changes in energy homeostasis and/or weight with bone mass, particularly within
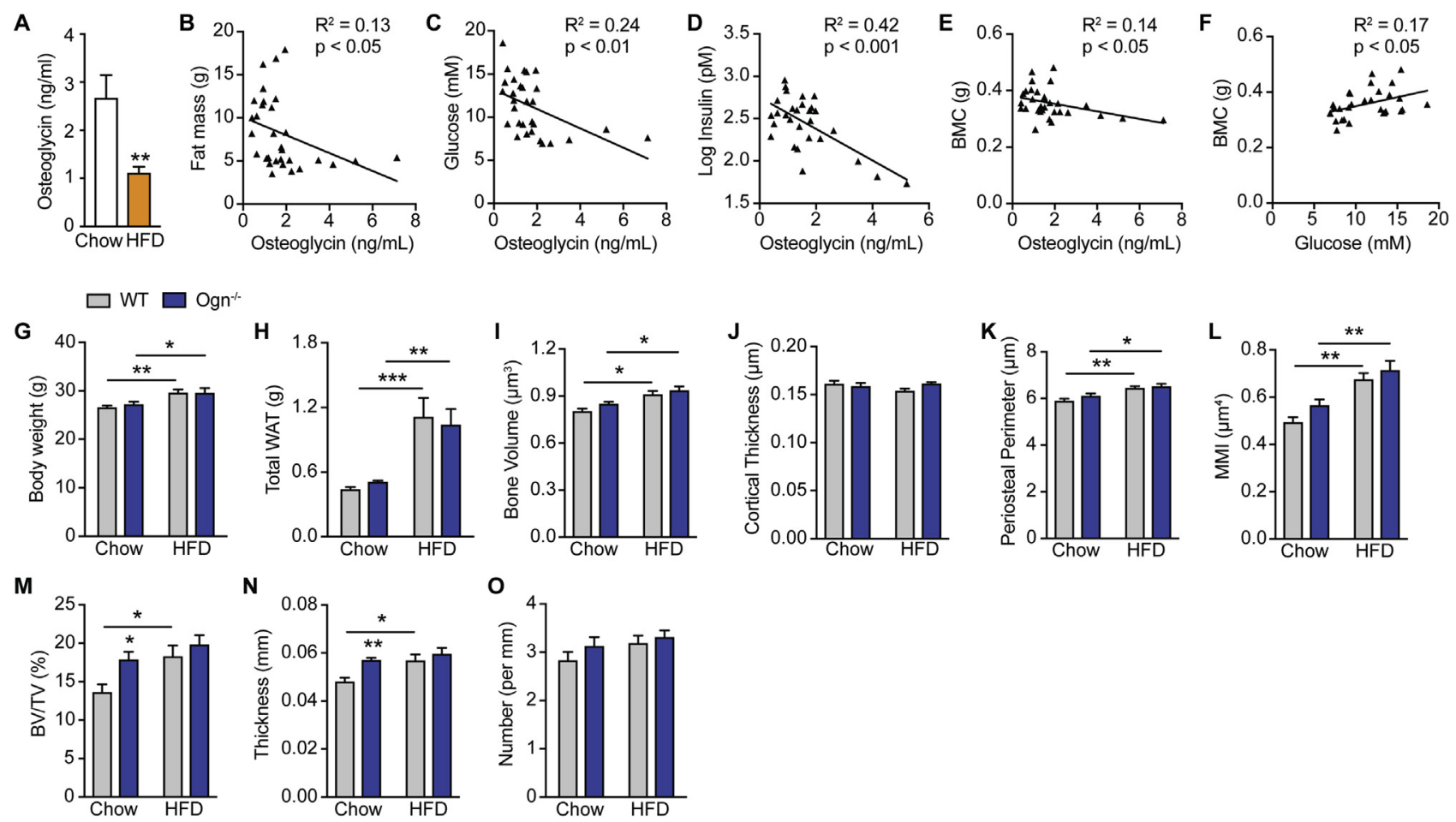

Figure 7: 0steoglycin negatively correlates with fat and bone mass in mice. (A) Osteoglycin levels at cull in wildtype (WT) mice fed either a chow or HFD. Correlations between osteoglycin levels and (B) fat mass, (C) glucose levels, (D) insulin levels and (E) whole body BMC as well as correlation between (F) glucose levels and whole body BMC in WT mice fed either chow or HFD. (G) Body weight and (H) total white adipose tissue (WAT) mass in WT and Ogn ${ }^{-1-}$ mice fed either a chow or HFD for 8 weeks. uCT analysis of the femora of WT or $\mathrm{Ogn}^{-1-}$ mice fed either a chow or HFD showing (I) cortical bone volume, (J) cortical thickness, (K) periosteal perimeter, (L) polar MMI, (M) trabecular bone volume (BV/TV), (N) trabecular thickness, and $(0)$ trabecular number. Data are means \pm SEM of at least 7 per group, ${ }^{*}=p<0.05,{ }^{\star \star}=p<0.01,{ }^{\star \star \star}=p<0.001$ as indicated. 
trabecular bone. The suppression of osteoglycin secretion during obesity acts to increase trabecular bone mass, enabling a compensatory increase in bone production during periods of weight gain.

\subsection{Osteoglycin response correlates with the glycemic response to weight loss in severely obese humans}

In order to explore whether osteoglycin acts to regulate blood glucose during periods of weight change in humans, we examined the changes in osteoglycin, weight, and glucose homeostasis in severely obese patients enrolled in a weight loss program involving several different weight loss interventions: dietary program, sleeve gastrectomy, and RouX-en-Y gastric bypass. Gastric surgery acts as a model of altered energy balance, producing a situation of negative energy balance, the contrasting model of diet-induced obesity, allowing a determination of whether osteoglycin is involved in the adaptation to both positive and negative energy balance. In the basal, pre-intervention state, there were no significant relationships between serum osteoglycin and either bodyweight or fat mass (Figure $8 \mathrm{~A}-\mathrm{B}$ ). However, there was a significant negative correlation between osteoglycin and BMI (Figure 8C) and a significant positive correlation between osteoglycin and lean mass (Figure 8D). Importantly, in the post-intervention period, significant associations between osteoglycin and energy and glucose homeostasis emerged. Consistent with osteoglycin reducing during weight gain in our mouse models, circulating osteoglycin levels increased following weight loss, particularly so in the patients with substantial weight loss as a result of gastric surgery. The increase in serum osteoglycin in the 1 month following intervention significantly correlated with weight loss (Figure 8E) and change in BMI (Figure 8F), with the least weight loss associated with the lowest change in osteoglycin levels. Moreover, the change in osteoglycin from baseline was significantly negatively correlated with fasting blood glucose (Figure 8G), whereby patients with a greater increase in osteoglycin levels displayed the lowest blood glucose levels. Thus, as with mice, osteoglycin in humans is associated with the control of glucose metabolism during times of altered energy balance.

\section{DISCUSSION}

Here we describe a novel regulatory circuitry between bone, pancreas, and the hypothalamus involved in the coordination of glucose homeostasis, bone formation, and energy balance. As a key component of this novel circuitry we identified osteoglycin as critical for mediating the adaptive response in bone to changing energy balance. Central NPY signaling coordinates global energy balance and bone formation by stimulating bone formation during positive energy balance and suppressing bone formation during negative energy balance, adapting bone mass to changing body weight and ensuring mechanical competence of the skeleton [4]. Now we show that this central NPY initiated process leads to subsequent osteoblastic $\mathrm{Y} 1$ signaling increasing osteoglycin secretion when energy balance is low. Using an osteoglycin-deficient mouse model and osteoglycin treatment in wildtype mice, we show that osteoglycin acts to suppress osteoblast activity and hence bone formation and, in addition, feeds back to help increase whole body energy balance by acting centrally on hypothalamic NPY-ergic neurons to increase food intake as well as by improving glucose uptake through modulation of insulin secretion and insulin action at target tissue such as muscle. While osteoglycin is produced from various tissues, these and our previous observation that the bone and glucose homeostatic defects of the Y1 deficient osteoblast model could be corrected with wildtype bone marrow transfer clearly implicate osteoblasts as an essential component of this process. Conversely, the low levels of circulating osteoglycin seen during obesity act dually to both reduce food intake and reduce insulin responsiveness, glucose uptake, and, as a consequence, increase blood glucose. The shift in blood glucose in turn increases the energy available to bone formation through increased glucose uptake into early osteoblasts, further enabling the skeleton to adapt to increases in body weight. This $Y 1$ receptor/osteoglycin axis highlights the complex interplay required to fine tune homeostatic requirements across multiple organ systems, and the importance of bone, as a weight bearing tissue, in this regulatory interplay. Importantly, the actions of
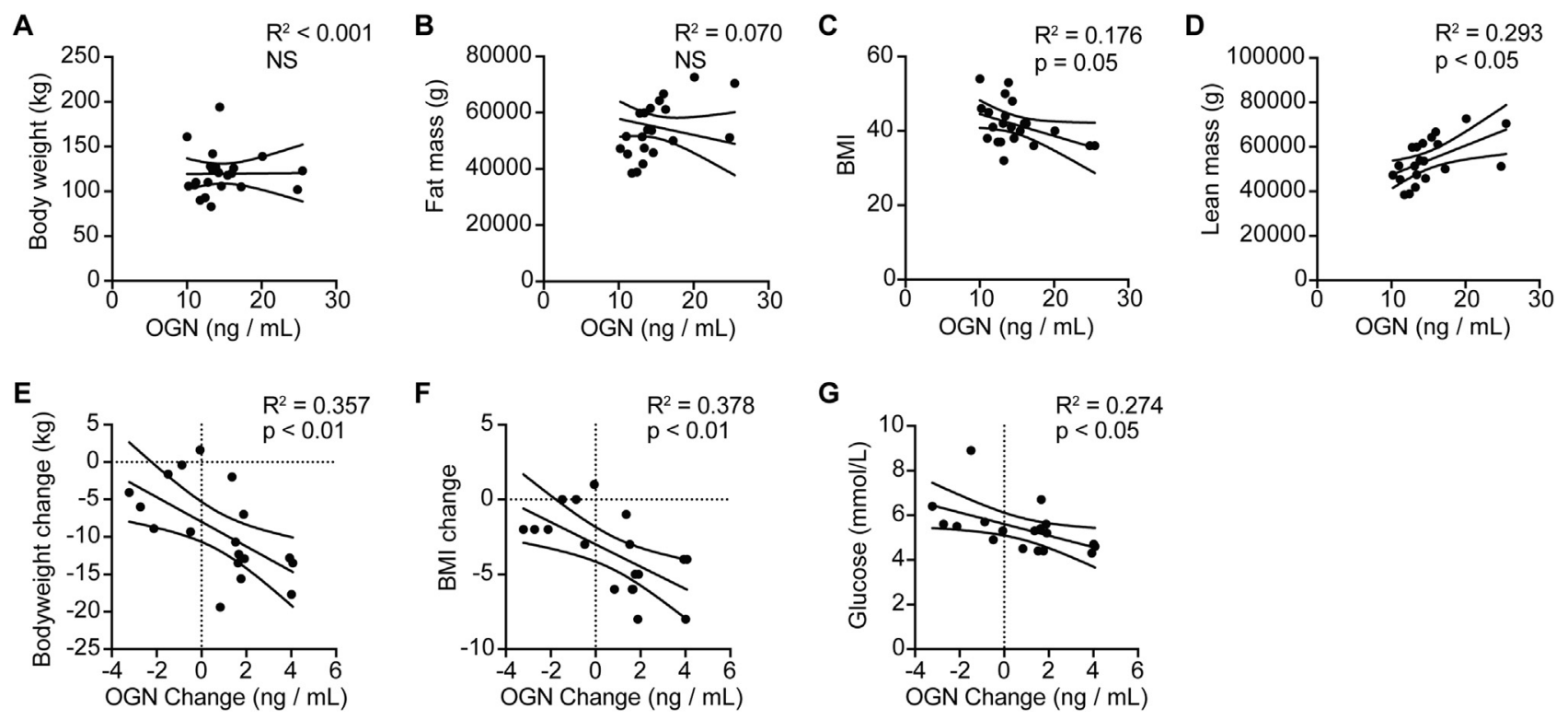

Figure 8: 0steoglycin response correlates with the glycemic response to weight loss in severely obese humans. Baseline correlations between osteoglycin levels and (A) body weight, (B) fat mass, (C) BMl, and (D) lean mass in severely obese patients in a weight loss intervention study. Correlations between the change in osteoglycin levels from preoperative state to 1 month post-intervention with (E) bodyweight change, (F) BMl change and (G) glucose levels at 1 month post-intervention. Data are means \pm SEM of at least 19 per group, $\mathrm{p}$ values are as indicated. NS = not significant. 
osteoglycin were evident in human studies, suggesting that it may provide a novel platform for development of therapies for metabolic dysfunction and the associated glycemic and skeletal complications. Osteoglycin, also known as osteoinductive factor or mimecan, is a small leucine-rich proteoglycan that is secreted into the extracellular matrix and was initially isolated from bovine bone as an inducer of matrix mineralisation [32]. It has also been shown to have a role in regulating left ventricular mass and collagen fibrillogenesis [33,34]. In addition, previous in vitro studies support a role for osteoglycin as a humoral factor in the regulation of bone mass [35]. This study now demonstrates a novel role for osteoglycin in the regulation of wholebody glucose homeostasis through effects on insulin secretion and insulin action, since lack of osteoglycin in mice leads to reduced $\beta$-cell mass, impaired glucose tolerance, and decreased insulin responsiveness, a condition which is exacerbated under high fat diet conditions. While our in vitro results show that osteoglycin treatment can stimulate insulin secretion in pancreatic islet cells, acute treatment in mice actually lowered insulin levels throughout the glucose tolerance test. This reduction in glucose-stimulated insulin levels observed in vivo is consistent with a predominant effect of osteoglycin to improve insulin action and glucose disposal, as supported by the ability of osteoglycin treatment to increase the response to an insulin tolerance test as well as synergistically enhance insulin-induced Akt phosphorylation in muscle. The ability of circulating osteoglycin to improve insulin sensitivity in target organs, as well as stimulate insulin release directly from the pancreas, highlights the multi-modal nature of its signaling axis.

It is now clear that bone, like other organs, is not only the recipient of hormonal signals but is also capable of transmitting signals which are then integrated into the regulation of many physiological processes. These results add osteoglycin as a novel factor to the previously identified osteocalcin [27] and lipocalin 2 [28] that have been implicated as being involved in the communication between bone and other tissues in the regulation of energy metabolism. While we acknowledge that osteoglycin is secreted from various tissue sources, some or all of which may be contributing to the phenotype observed in global $\mathrm{Ogn}^{-1-}$ mice, our data show that osteoglycin produced from osteoblastic cells is capable of stimulating insulin secretion directly from pancreatic beta cells. Furthermore, reduced osteoglycin secretion from osteoblasts in mice with osteoblast-specific Y1 receptor deletion is likely the mechanism behind their altered glucose homeostasis as bone marrow chimeric experiments confirms the osteoblastic nature of this phenotype [7]. Therefore, these data clearly support a role for bone-derived osteoglycin in the regulation of glucose homeostasis. However, we chose to study the effects of global osteoglycin deficiency to elucidate the effects of altering osteoglycin at the whole body level as it is more therapeutically relevant. The effects in $O g n^{-1-}$ mice were mirrored by osteoglycin treatment, which was able to increase glucose tolerance and insulin action, with a single injection. Thus, targeting osteoglycin pathways as a therapeutic may provide a platform for the development of novel treatments for patients with type 2 diabetes.

This study is the first to demonstrate the in vivo effects of osteoglycin on the regulation of bone mass. Previous in vitro studies have suggested that osteoglycin may suppress the differentiation of premature osteoblasts, at least partly by decreasing the levels of Runx2 and Osterix, but enhance the phenotype and mineralisation of mature osteoblasts [35]. Here we show that osteoglycin deficiency in mice led to an anabolic bone phenotype with significant increases in femur length, cancellous bone mass and cortical bone volume. This anabolic phenotype evident in $\mathrm{Ogn}^{-1-}$ mice is associated with marked increases in both mineral apposition rate and bone formation rate, suggesting enhanced osteoblast activity and confirming a role for osteoglycin in the suppression of osteoblast activity in vivo. In addition, osteoglycin may be acting to enhance osteoclastogenesis and bone resorption as evident by a significant decrease in osteoclast number in $\mathrm{Ogn}^{-1-}$ mice, consistent with the increase in bone mass observed. In addition to effects upon insulin and bone, osteoglycin also demonstrated signaling in the hypothalamus and the ability to regulate whole body energy homeostasis centrally. Osteoglycin deficient mice displayed a reduction in NPY expression in the hypothalamus and a consistent reduction in food intake. Conversely, osteoglycin treatment resulted in an increase in food intake, altered respiratory quotient (fuel utilisation), and direct neuronal activation in the hypothalamus, targeting NPY neurons directly but possibly also indirectly. Together these data suggest that osteoglycin mediates its effects on food intake via central NPY signaling.

In humans, our data suggest that circulating osteoglycin plays a role in regulating the response to a change in energy balance. We demonstrate that higher circulating osteoglycin levels correlate with both lower BMI and greater lean mass in severely obese patients and that weight loss induced by gastric surgery, increases serum osteoglycin. These data are consistent with our mouse studies which show that obesity reduces circulating levels of osteoglycin. Importantly, the change in osteoglycin levels from baseline to 1 month after weight loss intervention was associated with improved glucose levels, consistent with the action of osteoglycin to regulate glucose homeostasis, subsequent to a change in energy homeostasis, whereby change in energy balance alters osteoglycin, which then alters glucose balance.

Together, these data indicate that osteoglycin functions in humans as it does in mice, to regulate insulin sensitivity in accordance with energy balance to aide skeletal adaptation to energy/weight change. The suppression of osteoglycin secretion by bone cells during weight gain due to reduced NPY signaling would therefore represent a mechanism to diminish insulin action in order to suppress energy uptake in insulin target tissues. Such a change presents multiple advantages for bone. Firstly, the reduction in insulin action would increase blood glucose, a fundamental substrate for bone formation [36] particularly in early osteoblasts [31] rather than later, when free fatty acids may be more important [37]. This bias in glucose utilisation toward early osteoblasts is consistent with the ability of NPY/Y1 receptors to regulate glucose homeostasis through osteoglycin release also being restricted to early, not late osteoblasts [7], and suggests that osteoblast-mediated regulation of osteoglycin occurs in early osteoblasts, in line with its peak expression at this stage of differentiation [38]. The second advantage for bone from a reduction in insulin action is a shift in glucose disposal away from insulin-mediated glut4 pathways, such as muscle, toward glut1 and glut3, which are the principal transporters of glucose in the osteoblast [36]. In addition to energy provision in the form of glucose, in early osteoblasts, glut1 activity is pivotal to production of osteoblastic cells and subsequent formation of bone. Glut1-mediated glucose transport has been shown to trigger the expression of Runx2, the major osteoblast transcription factor [31]. Stable overexpression of osteoglycin in an osteoblast cell line or in conditioned media reduced Runx2 levels, while osteoglycin siRNA increased Runx2 [35]. Thus reducing serum osteoglycin would increase glut1 traffic and promote osteoblast differentiation and bone production via Runx2, enhancing the functional change in bone formation required for adaptive responses.

Taken together, these data identify osteoglycin as a circulating factor capable of regulating insulin action, bone mass and food intake. We show here that osteoglycin secretion is the downstream mediator of NPY signaling through Y1 receptors on early osteoblasts which serves 
as a component of the adaptation process acting to coordinate bone mass with global energy balance, thereby ensuring mechanical competence of the skeleton as weight increases and conserving energy when weight decreases. This adaptation process manifests as a reduction in osteoglycin secretion and subsequent suppression of glucose uptake in insulin target tissues through reduced insulin action when energy balance is positive. Conversely, when energy balance is negative, osteoglycin acts to suppress bone accrual and increase global glucose uptake while also feeding back centrally to stimulate food intake and increase energy balance. Thus, the actions of osteoglycin represent an adaptive process, which is highly integrated across multiple peripheral and central signaling cascades. Osteoglycin therefore emerges as an example of the sophisticated interplay required to coordinate tissue-specific energy requirements during times of global energy changes and the particular challenges faced by weight bearing systems such as the skeleton.

\section{CONFLICT OF INTEREST}

None declared.

\section{APPENDIX A. SUPPLEMENTARY DATA}

Supplementary data related to this article can be found at https://doi.org/10.1016/j. molmet.2018.05.004.

\section{REFERENCES}

[1] Loh, K., Herzog, H., Shi, Y.C., 2015. Regulation of energy homeostasis by the NPY system. Trends in Endocrinology and Metabolism 26(3):125-135.

[2] Baldock, P.A., Sainsbury, A., Couzens, M., Enriquez, R.F., Thomas, G.P., Gardiner, E.M., et al., 2002. Hypothalamic Y2 receptors regulate bone formation. Journal of Clinical Investigation 109(7):915-921.

[3] Lee, N.J., Doyle, K.L., Sainsbury, A., Enriquez, R.F., Hort, Y.J., Riepler, S.J., et al., 2010. Critical role for Y1 receptors in mesenchymal progenitor cell differentiation and osteoblast activity. Journal of Bone and Mineral Research 25(8):1736-1747.

[4] Baldock, P.A., Lee, N.J., Driessler, F., Lin, S., Allison, S., Stehrer, B., et al., 2009. Neuropeptide $Y$ knockout mice reveal a central role of NPY in the coordination of bone mass to body weight. PLoS One 4(12):e8415.

[5] Lee, N.J., Nguyen, A.D., Enriquez, R.F., Doyle, K.L., Sainsbury, A., Baldock, P.A., et al., 2011. Osteoblast specific Y1 receptor deletion enhances bone mass. Bone 48(3):461-467.

[6] Matic, I., Matthews, B.G., Kizivat, T., Igwe, J.C., Marijanovic, I., Ruohonen, S.T., et al., 2012. Bone-specific overexpression of NPY modulates osteogenesis. Journal of Musculoskeletal and Neuronal Interactions 12(4):209-218.

[7] Lee, N.J., Nguyen, A.D., Enriquez, R.F., Luzuriaga, J., Bensellam, M., Laybutt, R., et al., 2015. NPY signaling in early osteoblasts controls glucose homeostasis. Molecular Metabolism 4(3):164-174.

[8] Kalajzic, I., Kalajzic, Z., Kaliterna, M., Gronowicz, G., Clark, S.H., Lichtler, A.C., et al., 2002. Use of type I collagen green fluorescent protein transgenes to identify subpopulations of cells at different stages of the osteoblast lineage. Journal of Bone and Mineral Research 17(1):15-25.

[9] Howell, O.W., Scharfman, H.E., Herzog, H., Sundstrom, L.E., BeckSickinger, A., Gray, W.P., 2003. Neuropeptide $Y$ is neuroproliferative for postnatal hippocampal precursor cells. Journal of Neurochemistry 86(3):646-659.

[10] Yang, H., Wang, H., Jaenisch, R., 2014. Generating genetically modified mice using CRISPR/Cas-mediated genome engineering. Nature Protocols 9(8):1956-1968.

[11] Ran, F.A., Hsu, P.D., Lin, C.Y., Gootenberg, J.S., Konermann, S., Trevino, A.E., et al., 2013. Double nicking by RNA-guided CRISPR Cas9 for enhanced genome editing specificity. Cell 154(6):1380-1389.
[12] Yu, E.W., Bouxsein, M.L., Putman, M.S., Monis, E.L., Roy, A.E., Pratt, J.S., et al., 2015. Two-year changes in bone density after Roux-en-Y gastric bypass surgery. Journal of Clinical Endocrinology \& Metabolism 100(4):1452-1459.

[13] Awazawa, M., Ueki, K., Inabe, K., Yamauchi, T., Kubota, N., Kaneko, K., et al., 2011. Adiponectin enhances insulin sensitivity by increasing hepatic IRS-2 expression via a macrophage-derived IL-6-dependent pathway. Cell Metabolism 13(4):401-412.

[14] Maghrabi, A.H., Wolski, K., Abood, B., Licata, A., Pothier, C., Bhatt, D.L., et al., 2015. Two-year outcomes on bone density and fracture incidence in patients with T2DM randomized to bariatric surgery versus intensive medical therapy. Obesity 23(12):2344-2348.

[15] Irizarry, R.A., Bolstad, B.M., Collin, F., Cope, L.M., Hobbs, B., Speed, T.P., 2003. Summaries of Affymetrix GeneChip probe level data. Nucleic Acids Research 31(4):e15.

[16] Gautier, L., Cope, L., Bolstad, B.M., Irizarry, R.A., 2004. Affy-analysis of Affymetrix GeneChip data at the probe level. Bioinformatics 20(3):307-315.

[17] Gentleman, R.C., Carey, V.J., Bates, D.M., Bolstad, B., Dettling, M., Dudoit, S., et al., 2004. Bioconductor: open software development for computational biology and bioinformatics. Genome Biology 5(10):R80.

[18] Ihaka, R., Gentleman, R.C., 1996. R: a language for data analysis and graphics. Journal of Computational \& Graphical Statistics 5:299-314.

[19] Smyth, G.K., 2004. Linear models and empirical bayes methods for assessing differential expression in microarray experiments. Statistical Applications in Genetics and Molecular Biology 3. Article3.

[20] Subramanian, A., Tamayo, P., Mootha, V.K., Mukherjee, S., Ebert, B.L., Gillette, M.A., et al., 2005. Gene set enrichment analysis: a knowledge-based approach for interpreting genome-wide expression profiles. Proceedings of the National Academy of Sciences 102(43):15545-15550.

[21] Reich, M., Liefeld, T., Gould, J., Lerner, J., Tamayo, P., Mesirov, J.P., 2006. GenePattern 2.0. Nature Genetics 38(5):500-501.

[22] Shevchenko, A., Tomas, H., Havlis, J., Olsen, J.V., Mann, M., 2006. In-gel digestion for mass spectrometric characterization of proteins and proteomes. Nature Protocols 1(6):2856-2860.

[23] Rappsilber, J., Mann, M., Ishihama, Y., 2007. Protocol for micro-purification, enrichment, pre-fractionation and storage of peptides for proteomics using StageTips. Nature Protocols 2(8):1896-1906.

[24] Cox, J., Neuhauser, N., Michalski, A., Scheltema, R.A., Olsen, J.V., Mann, M., 2011. Andromeda: a peptide search engine integrated into the MaxQuant environment. Journal of Proteome Research 10(4):1794-1805.

[25] Zhang, L., Macia, L., Turner, N., Enriquez, R.F., Riepler, S.J., Nguyen, A.D., et al., 2010. Peripheral neuropeptide Y Y1 receptors regulate lipid oxidation and fat accretion. International Journal of Obesity 34(2):357-373.

[26] Baldock, P.A., Sainsbury, A., Allison, S., Lin, E.J., Couzens, M., Boey, D., et al., 2005. Hypothalamic control of bone formation: distinct actions of leptin and y2 receptor pathways. Journal of Bone and Mineral Research 20(10): $1851-1857$.

[27] Lee, N.K., Sowa, H., Hinoi, E., Ferron, M., Ahn, J.D., Confavreux, C., et al., 2007. Endocrine regulation of energy metabolism by the skeleton. Cell 130(3):456-469.

[28] Mosialou, I., Shikhel, S., Liu, J.M., Maurizi, A., Luo, N., He, Z., et al., 2017. MC4R-dependent suppression of appetite by bone-derived lipocalin 2. Nature 543(7645):385-390.

[29] Jiang, Z.Y., Zhou, Q.L., Coleman, K.A., Chouinard, M., Boese, Q., Czech, M.P., 2003. Insulin signaling through Akt/protein kinase B analyzed by small interfering RNA-mediated gene silencing. Proceedings of the National Academy of Sciences of the U S A 100(13):7569-7574.

[30] Cao, H.M., Ye, X.P., Ma, J.H., Jiang, H., Li, S.X., Li, R.Y., et al., 2015. Mimecan, a hormone abundantly expressed in adipose tissue, reduced food intake independently of leptin signaling. EBioMedicine 2(11):1718-1724.

[31] Wei, J., Shimazu, J., Makinistoglu, M.P., Maurizi, A., Kajimura, D., Zong, H., et al., 2015. Glucose uptake and Runx2 synergize to orchestrate osteoblast differentiation and bone formation. Cell 161(7):1576-1591. 
[32] Bentz, H., Nathan, R.M., Rosen, D.M., Armstrong, R.M., Thompson, A.Y., Segarini, P.R., et al., 1989. Purification and characterization of a unique osteoinductive factor from bovine bone. Journal of Biological Chemistry 264(34):20805-20810.

[33] Ge, G., Seo, N.S., Liang, X., Hopkins, D.R., Hook, M., Greenspan, D.S., 2004. Bone morphogenetic protein-1/tolloid-related metalloproteinases process osteoglycin and enhance its ability to regulate collagen fibrillogenesis. Journal of Biological Chemistry 279(40):41626-41633.

[34] Tasheva, E.S., Koester, A., Paulsen, A.Q., Garrett, A.S., Boyle, D.L., Davidson, H.J., et al., 2002. Mimecan/osteoglycin-deficient mice have collagen fibril abnormalities. Molecular Vision 8:407-415.

[35] Tanaka, K., Matsumoto, E., Higashimaki, Y., Katagiri, T., Sugimoto, T., Seino, S., et al., 2012. Role of osteoglycin in the linkage between muscle and bone. Journal of Biological Chemistry 287(15):11616-11628.
[36] Lee, W.C., Guntur, A.R., Long, F., Rosen, C.J., 2017. Energy metabolism of the osteoblast: implications for osteoporosis. Endocrine Reviews 38(3):255-266.

[37] Kushwaha, P., Wolfgang, M.J., Riddle, R.C., 2017. Fatty acid metabolism by the osteoblast. Bone.

[38] Paic, F., Igwe, J.C., Nori, R., Kronenberg, M.S., Franceschetti, T., Harrington, P., et al., 2009. Identification of differentially expressed genes between osteoblasts and osteocytes. Bone 45(4):682-692.

\section{FURTHER READING}

[39] Karl, T., Duffy, L., Herzog, H., 2008. Behavioural profile of a new mouse model for NPY deficiency. European Journal of Neuroscience 28(1):173-180.

[40] Loh, K., Zhang, L., Brandon, A., Wang, Q., Begg, D., Qi, Y., etal., 2017. Insulin controls food intake and energy balance via NPY neurons. Mol Metab 6(6):574-584. 